\title{
Leçons tirées de dix pays sur la réglementation des antimicrobiens pour les animaux d'élevage
}

\author{
Prativa Baral (D), Marie E. Danik and Steven J. Hoffman
}

\section{Résumé}

Cette étude examine les approches réglementaires de dix pays : la France, le Danemark, l'Australie, le Canada, les États-Unis, la Russie, le Japon, le Brésil, la Chine et l'Inde, relativement à la réglementation des antimicrobiens aux trois points d'entrée principaux dans le processus agricole, soit dans le cadre de la médecine vétérinaire, de la production agricole et de la vente des produits agricoles. Pour être efficace, la réglementation doit tenir compte du contexte dans lequel elle s'inscrit. Cette étude tente donc de tirer des leçons des dix juridictions ci-haut à la lumière des réalités qui entourent l'utilisation d'antimicrobiens dans les processus agricoles.

Mots clés : antimicrobiens, résistance antimicrobienne, réglementations, animaux d'élevage, approches réglementaires internationales

\begin{abstract}
This study examines the regulatory approaches of ten countries: France, Denmark, Australia, Canada, the United States, Russia, Japan, Brazil, China and India with regard to the regulation of antimicrobials at the three main points of entry into the agricultural process, that is in veterinary medicine, in agricultural production, and in the sale of agricultural products. To be effective, regulation must take into account the context in which it operates. This study therefore attempts to draw lessons from the ten jurisdictions above, in the light of their realities around the use of antimicrobials in the agricultural process.
\end{abstract}

Keywords: antimicrobials, antimicrobial resistance, regulations, farm animals, international regulatory approaches

\section{Introduction}

L'accroissement rapide des taux de résistance antimicrobienne est un problème de santé publique urgent et d'importance considérable. Malgré cela, certains pays tardent à adopter des règles pour encadrer l'utilisation des antimicrobiens ou adoptent des

Canadian Journal of Law and Society / Revue Canadienne Droit et Société, 2019. This is an Open Access article, distributed under the terms of the Creative Commons AttributionNonCommercial-ShareAlike licence (http://creativecommons.org/licenses/by-ncsa/ 4.0/), which permits non-commercial re-use, distribution, and reproduction in any medium, provided the same Creative Commons licence is included and the original work is properly cited. The written permission of Cambridge University Press must be obtained for commercial re-use.

Volume 34, no. 3, pp. 521-553. doi:10.1017/cls.2019.25 
stratégies qui ne touchent pas à toutes les dimensions de la problématique. Dans le but de déceler certains des défis qui peuvent retarder l'adoption de telles règles et d'identifier des incitations qui pourraient encourager certains pays à passer à l'action, cette étude examine les approches réglementaires de dix pays : la France, le Danemark, l'Australie, le Canada, les États-Unis, la Russie, le Japon, le Brésil, la Chine et l'Inde.

Plus précisément, cette étude se penche sur la réglementation aux trois points d’entrée principaux dans le processus agricole : la médecine vétérinaire, la production agricole et la vente des produits agricoles. Ce faisant, elle met de l'avant les liens importants qui existent entre les diverses activités régies par ces domaines. Par exemple, il existe des liens importants entre : les approbations nécessaires pour la commercialisation des produits pharmaceutiques et le rôle des vétérinaires sur le plan de la surveillance, les produits antimicrobiens dont disposent les producteurs de bétail et les usages qu'ils peuvent en faire, ainsi que les exigences en matière de salubrité des aliments et aux résidus antimicrobiens dans les produits destinés au marché intérieur, en comparaison avec ceux destinés aux marchés extérieurs.

De nombreux défis à la réglementation sont ainsi soulevés, puisque cette multidisciplinarité pose une difficulté particulière : les interrelations entre les sphères d'activités qui contribuent à la propagation de résistances contre les pathogènes créent un système dynamique qui doit être géré dans son ensemble. Cette approche intégrée, communément appelée «One Health ${ }^{1}$ » (ou « Une seule santé »), est donc nécessaire pour aborder les dynamiques qui sont au cœur de la problématique.

Tout cela peut poser un véritable casse-tête aux décideurs puisqu'ils doivent tenir compte de cette multitude d'enjeux et d'acteurs et, par conséquent, doivent envisager l'adoption de modifications règlementaires coordonnées dans plusieurs domaines et instruments à la fois. De plus, en raison des nombreux liens qui existent entre les sphères d'activités au cœur de cette problématique, le résultat des changements règlementaires peut être difficile à prévoir. Par exemple, une règlementation qui a eu beaucoup de succès dans un pays pourrait s'avérer inefficace dans un autre contexte. Ainsi, la règlementation doit être adaptée au contexte local.

Cette étude permet donc d'identifier certaines interdépendances clés et l'importance relative accordée par ces juridictions aux facteurs nommés suivant leur contexte. Elle s'effectue sur trois plans (Figure 1). Premièrement, la réglementation de la médecine vétérinaire est examinée. Cette étape préliminaire est un aspect clé du processus règlementaire puisqu’elle détermine de quels antimicrobiens les agriculteurs peuvent disposer en établissant des exigences pour leur mise en marché et leur distribution. Deuxièmement, le cadre réglementaire de l'utilisation des agents antimicrobiens dans la production agricole des animaux d'élevage, et notamment le bétail, sera abordé. Cela porte spécifiquement sur les usages permis et interdits des agents antimicrobiens pour les animaux d'élevage. Troisièmement, les règlements qui régissent le commerce intérieur et extérieur des produits animaliers destinés à la consommation seront discutés, afin de mieux cerner les considérations plus larges qui sont souvent déterminantes en matière de politique publique.

OIE Bulletin, «The 'One health' concept: the OIE approach » (2013), p. $1<$ http://www.oie.int/ fileadmin/Home/eng/Publications_\%26_Documentation/docs/pdf/bulletin/Bull_2013-1ENG.pdf $>$. 


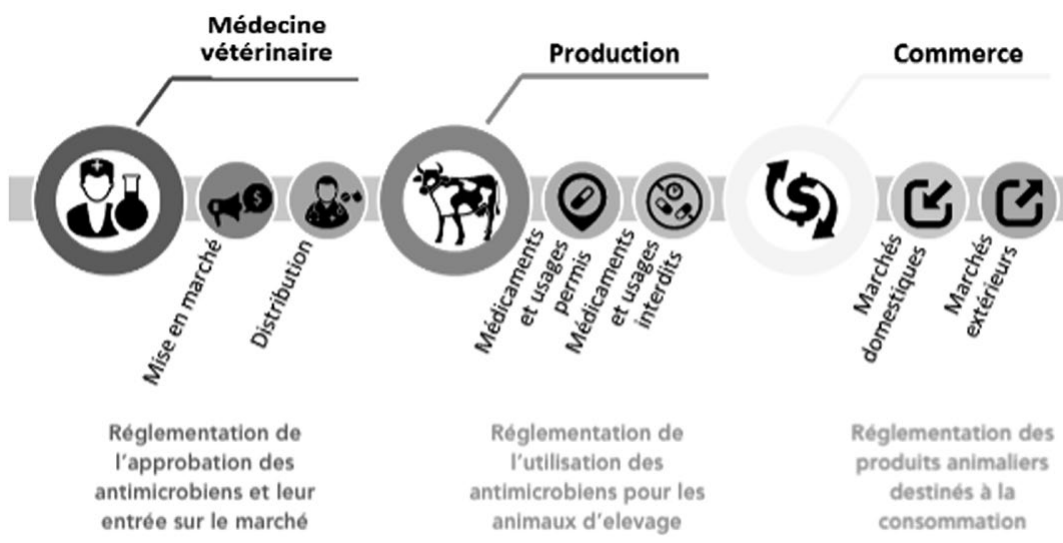

Figure 1 Points d'entrée des antimicrobiens au cours du processus agricole. Les pictogrammes dans les figures 1 et 2 proviennent du Noun Project (thenounproject.com), un site Internet dont le contenu est sous la licence Creative Commons. Les auteurs des pictogrammes, en ordre d'apparition, de gauche à droite, sont Alex Auda Samora, Gregor Črešnar, Wilson Joseph, James Keuning, lastspark, GRACE Communications Foundation and Mother Jones, Artur Shageyev, et doorfortyfour

Certaines constatations s'imposent alors. Entre autres, nous remarquons la grande efficacité de la réglementation qui agit sur le plan de la médecine vétérinaire. Les vétérinaires jouent alors un rôle clé et, en l'absence de solutions qui remplacent les antimicrobiens, il peut être difficile de trouver un équilibre entre deux impératifs de santé publique : assurer la santé des animaux et préserver l'utilité des antimicrobiens. Effectivement, sur le plan de la production agricole, les antibiotiques sont parfois utilisés pour pallier des lacunes du système de production, notamment au niveau de la salubrité. Les usages permis varient donc grandement d'une juridiction à l'autre, ce qui peut entraver l'accès de certains producteurs à des marchés importants. Des marchés « à deux vitesses » risquent alors de s'installer dans les pays dont les ressources sont limitées.

Cette étude est donc une première tentative pour conceptualiser des pistes d'amélioration face au défi mondial de la résistance antimicrobienne en utilisant des règlements réels et en vigueur dans dix juridictions.

\section{Méthodologie}

Cette étude vise à approfondir la discussion de trois façons. Premièrement, elle identifie les approches réglementaires concernent les politiques relatives à la médecine vétérinaire, la production agricole et le commerce. Deuxièmement, elle discute les tendances de règlementation de la résistance antimicrobienne établies et à venir. Troisièmement, elle permet de surmonter les principaux obstacles à la réglementation, ainsi que les incitations qui pourraient être déterminantes pour passer à l'action. En tout, cette étude permet d'identifier certaines interdépendances clés qui pourraient améliorer l'efficacité des règlements adoptés.

Les données ont été recueillies auprès de sources premières et secondaires, de textes scientifiques révisés par des pairs, des textes de littérature grise, des communiqués de presse, ainsi que des entrevues d'experts et de représentants 


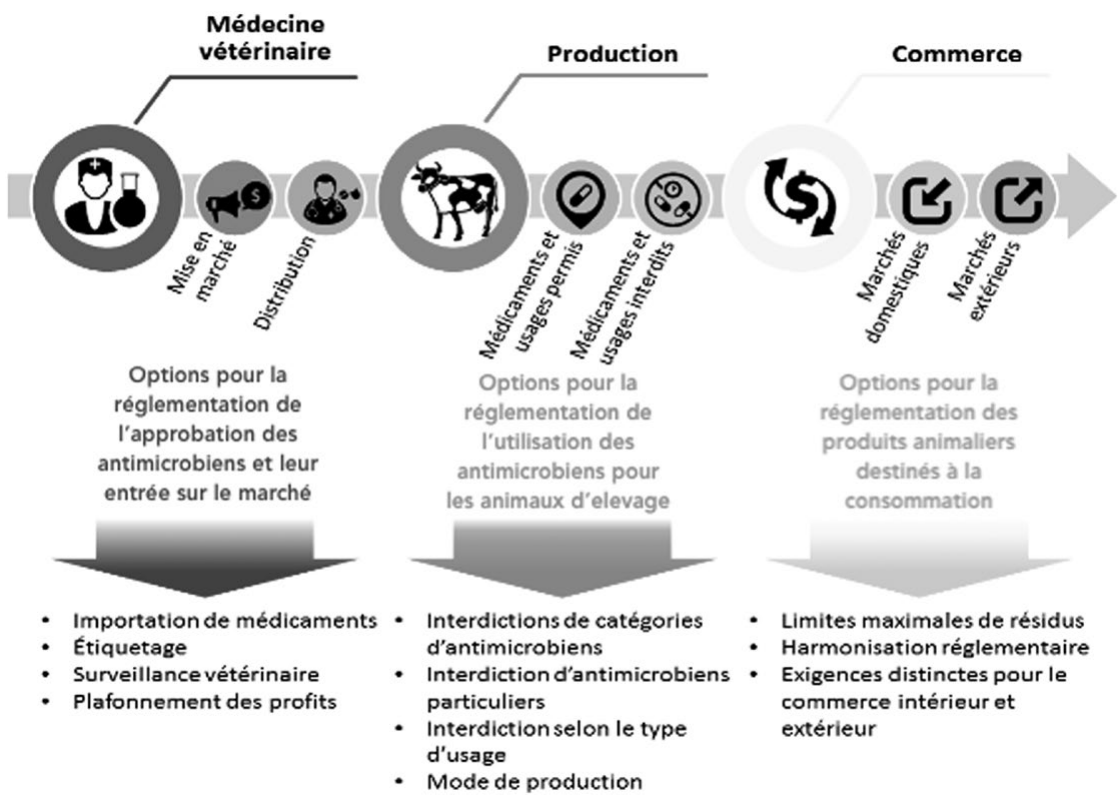

Figure 2 Options pour la réglementation des antimicrobiens aux trois points d’entrée principaux

d'État publiées par les médias. Les dix pays énumérés ci-dessus représentent un échantillon choisi à dessein. Ils ont été identifiés en fonction de trois critères : leur part respective des marchés mondiaux de l'importation et de l'exportation du bétail, ainsi que, dans l'ensemble, leur représentativité des différentes approches réglementaires possibles et des différentes régions géographiques.

\section{Limites}

Cette étude comporte certaines limites qui pourraient jouer sur l'application des résultats $^{2}$. Les données disponibles ${ }^{3}$ ne tiennent pas compte de certains facteurs clés pour appuyer l'analyse comparative, notamment le type et la quantité d'antibiotiques utilisés dans chaque pays, ainsi que les fins auxquels ils sont utilisés. Le manque de données à cet égard est un problème répandu dans ce domaine ${ }^{4}$.

2 Le manque de données sur le type et la quantité d’antibiotiques utilisés dans chaque pays, ainsi que les fins auxquelles ils sont utilisés, est un problème répandu dans ce domaine. De plus, létude de l'efficacité des règlements tient difficilement compte de l'usage réel en raison de failles au niveau de la surveillance. La délimitation du sujet de recherche limite également lapplication des conclusions. Cette étude est axée sur l'agriculture du cheptel vif et plus particulièrement, l'élevage du bétail (la volaille est donc exclue). La réglementation varie beaucoup en fonction du type d'animal élevé, entre autres, en raison des modes de production et des antimicrobiens particuliers qui sont nécessaires pour traiter différents animaux.

3 Les données ont été recueillies d’après des sources premières et secondaires, des textes scientifiques révisés par des pairs, des textes de littérature grise, des communiqués de presse, ainsi que des entrevues d'experts et de représentants étatiques publiées dans les médias.

4 Peter Collignon et Andreas Voss, "China, what antibiotics and what volumes are used in food production animals? (éditorial) ", Antimicrobial Resistance and Infection Control 4 (2015): 16 doi: 10.1186/s13756-015-0056-5. 


\section{Tableau I}

Tableau récapitulatif des résultats

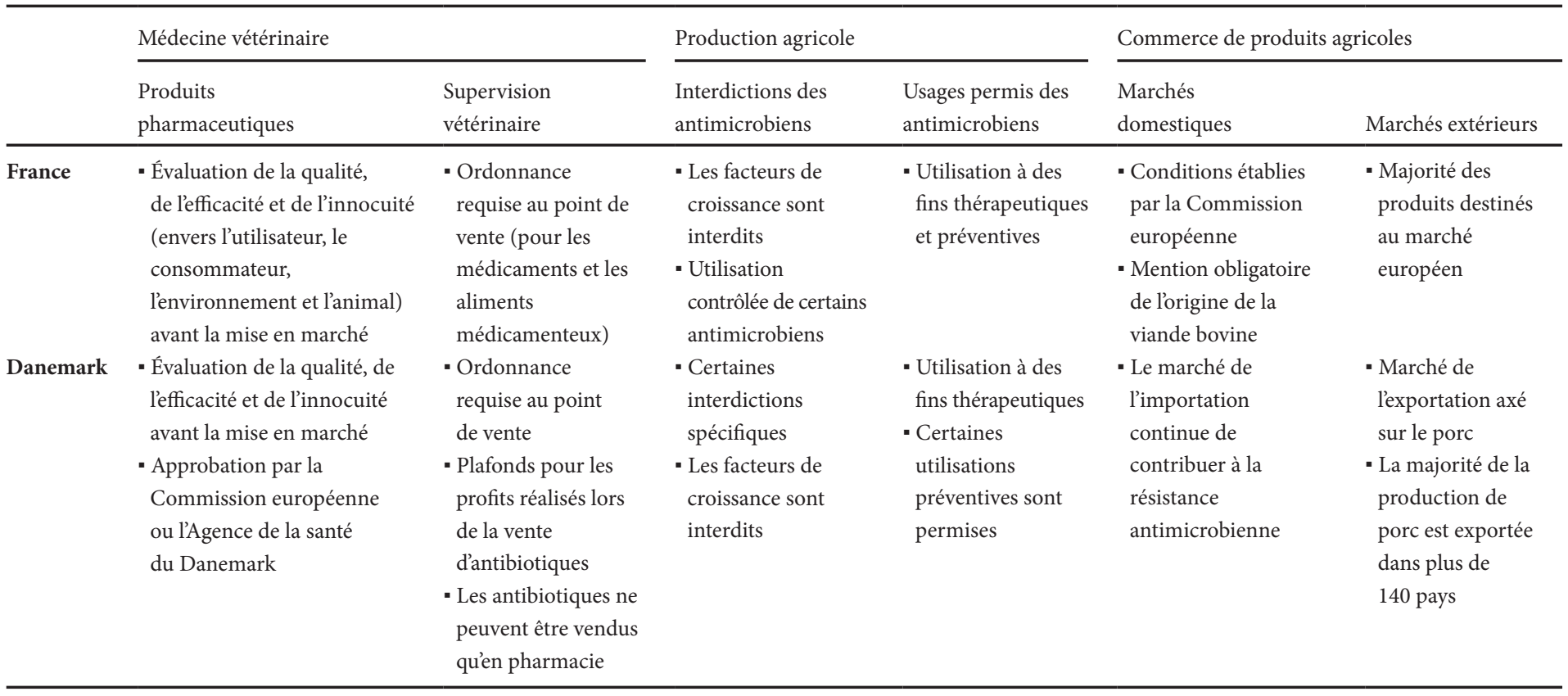




\begin{tabular}{|c|c|c|c|c|c|c|}
\hline & \multicolumn{2}{|l|}{ Médecine vétérinaire } & \multicolumn{2}{|l|}{ Production agricole } & \multicolumn{2}{|c|}{ Commerce de produits agricoles } \\
\hline & $\begin{array}{l}\text { Produits } \\
\text { pharmaceutiques }\end{array}$ & $\begin{array}{l}\text { Supervision } \\
\text { vétérinaire }\end{array}$ & $\begin{array}{l}\text { Interdictions des } \\
\text { antimicrobiens }\end{array}$ & $\begin{array}{l}\text { Usages permis des } \\
\text { antimicrobiens }\end{array}$ & $\begin{array}{l}\text { Marchés } \\
\text { domestiques }\end{array}$ & Marchés extérieurs \\
\hline Australie & $\begin{array}{l}\text { - Contraintes au niveau } \\
\text { de l'étiquetage } \\
\text { - Processus d’approbation } \\
\text { des médicaments très } \\
\text { conservateur }\end{array}$ & $\begin{array}{l}\text { - Ordonnance requise } \\
\text { au point de vente } \\
\text { - Certaines } \\
\text { variations du } \\
\text { "off-label use }{ }^{*} \text { selon } \\
\text { létat ou le territoire }\end{array}$ & $\begin{array}{l}\text { - Certaines } \\
\text { interdictions } \\
\text { spécifiques } \\
\text { - Utilisation contrôlée } \\
\text { des antimicrobiens } \\
\text { importants en santé } \\
\text { humaine }\end{array}$ & $\begin{array}{l}\text { - Utilisation à des } \\
\text { fins thérapeutiques, } \\
\text { préventives et } \\
\text { comme facteurs } \\
\text { de croissance }\end{array}$ & $\begin{array}{l}\text { - Surveillance des } \\
\text { antimicrobiens } \\
\text { en fonction des } \\
\text { volumes de vente }\end{array}$ & $\begin{array}{l}\text { - Quelques } \\
\text { différences avec } \\
\text { le marché } \\
\text { domestique } \\
\text { sur les résidus } \\
\text { permis }\end{array}$ \\
\hline Canada & $\begin{array}{l}\text { - Suggestions au niveau } \\
\text { de létiquetage }\end{array}$ & $\begin{array}{l}\text { - Les exigences } \\
\text { relatives aux } \\
\text { vétérinaires varient } \\
\text { selon la province }\end{array}$ & $\begin{array}{l}\text { - Utilisation contrôlée } \\
\text { de certains } \\
\text { antimicrobiens }\end{array}$ & $\begin{array}{l}\text { - Utilisation à des } \\
\text { fins thérapeutiques, } \\
\text { préventives et } \\
\text { comme facteurs } \\
\text { de croissance }\end{array}$ & $\begin{array}{l}\text { - Distinction législative } \\
\text { entre l'importation de } \\
\text { médicaments à des fins } \\
\text { commerciales et pour } \\
\text { l'utilisation personnelle }\end{array}$ & $\begin{array}{l}\text { - Le marché reprend } \\
\text { de l'essor après une } \\
\text { baisse au cours des } \\
\text { dernières années }\end{array}$ \\
\hline États-Unis & $\begin{array}{l}\text { - Suggestions au niveau } \\
\text { de létiquetage }\end{array}$ & $\begin{array}{l}\text { - Ordonnance } \\
\text { suggérée au point } \\
\text { de vente } \\
\text { - Certaines } \\
\text { restrictions sur } \\
\text { le «off-label use »* }\end{array}$ & $\begin{array}{l}\text { - Marché très } \\
\text { permissible } \\
\text { - Quelques } \\
\text { interdictions } \\
\text { spécifiques }\end{array}$ & $\begin{array}{l}\text { - Utilisation à des } \\
\text { fins thérapeutiques, } \\
\text { préventives et } \\
\text { comme facteurs } \\
\text { de croissance }\end{array}$ & $\begin{array}{l}\text { - Les exigences portent } \\
\text { notamment sur la } \\
\text { salubrité des aliments }\end{array}$ & $\begin{array}{l}\text { - Exigences plus } \\
\text { rigoureuses que } \\
\text { pour le marché } \\
\text { domestique }\end{array}$ \\
\hline
\end{tabular}

- Contraintes au niveau des médicaments très conservateur

Suggestions au niveau relatives aux vétérinaires varient suggérée au point de vente restrictions sur le « off-label use »* 
Tableau I. Continued

\begin{tabular}{|c|c|c|c|c|c|c|}
\hline & \multicolumn{2}{|l|}{ Médecine vétérinaire } & \multicolumn{2}{|l|}{ Production agricole } & \multicolumn{2}{|c|}{ Commerce de produits agricoles } \\
\hline & $\begin{array}{l}\text { Produits } \\
\text { pharmaceutiques }\end{array}$ & $\begin{array}{l}\text { Supervision } \\
\text { vétérinaire }\end{array}$ & $\begin{array}{l}\text { Interdictions des } \\
\text { antimicrobiens }\end{array}$ & $\begin{array}{l}\text { Usages permis des } \\
\text { antimicrobiens }\end{array}$ & $\begin{array}{l}\text { Marchés } \\
\text { domestiques }\end{array}$ & Marchés extérieurs \\
\hline Russie & $\begin{array}{l}\text { - Réformes réglementaires } \\
\text { générales sur la } \\
\text { surveillance, lapprobation, } \\
\text { l'enregistrement et la } \\
\text { circulation de médicaments } \\
\text { vétérinaires }\end{array}$ & $\begin{array}{l}\text { - Aucune } \\
\text { ordonnance } \\
\text { requise au point } \\
\text { de vente }\end{array}$ & $\begin{array}{l}\text { - Certaines } \\
\text { interdictions } \\
\text { spécifiques } \\
\text { - Utilisation contrôlée } \\
\text { de certains } \\
\text { antimicrobiens }\end{array}$ & $\begin{array}{l}\text { - Utilisation à des } \\
\text { fins thérapeutiques, } \\
\text { préventives et } \\
\text { comme facteurs } \\
\text { de croissance }\end{array}$ & $\begin{array}{l}\text { - Embargos et } \\
\text { restrictions } \\
\text { spécifiques }\end{array}$ & $\begin{array}{l}\text { - Changements } \\
\text { dans le contexte } \\
\text { de la production } \\
\text { domestique en } \\
\text { raison des } \\
\text { interdictions et } \\
\text { embargos }\end{array}$ \\
\hline Japon & $\begin{array}{l}\text { - Contraintes au niveau de } \\
\text { l'étiquetage } \\
\text { - Processus strict } \\
\text { d'approbation des } \\
\text { médicaments } \\
\text { - Normes de gestion du } \\
\text { risque harmonisées avec } \\
\text { les normes internationales }\end{array}$ & $\begin{array}{l}\text { - Ordonnance } \\
\text { requise au point } \\
\text { de vente } \\
\text { - Le « off-label } \\
\text { use »* est permis } \\
\text { sous la supervision } \\
\text { d'un vétérinaire }\end{array}$ & $\begin{array}{l}\text { - Utilisation } \\
\text { contrôlée de } \\
\text { certains } \\
\text { antimicrobiens } \\
\text { - Liste exhaustive } \\
\text { des additifs } \\
\text { alimentaires } \\
\text { permis }\end{array}$ & $\begin{array}{l}\text { - Utilisation à des } \\
\text { fins thérapeutiques, } \\
\text { préventives et } \\
\text { comme facteurs } \\
\text { de croissance }\end{array}$ & $\begin{array}{l}\text { - Contrôle très } \\
\text { rigoureux des } \\
\text { produits qui } \\
\text { entrent au pays }\end{array}$ & $\begin{array}{l}\text { - Rapport } \\
\text { commercial étroit } \\
\text { entre les } \\
\text { compagnies } \\
\text { importatrices et } \\
\text { les producteurs } \\
\text { (partage de } \\
\text { connaissances } \\
\text { techniques) }\end{array}$ \\
\hline Brésil & $\begin{array}{l}\text { - Réformes réglementaires } \\
\text { sur la vente et la } \\
\text { surveillance }\end{array}$ & $\begin{array}{l}\text { - Ordonnance } \\
\text { requise au point } \\
\text { de vente }\end{array}$ & $\begin{array}{l}\text { - Certaines } \\
\text { interdictions } \\
\text { spécifiques }\end{array}$ & $\begin{array}{l}\text { - Utilisation à des } \\
\text { fins thérapeutiques, } \\
\text { préventives et } \\
\text { comme facteurs } \\
\text { de croissance }\end{array}$ & $\begin{array}{l}\text { - Marché axé sur la } \\
\text { production et } \\
\text { l'exportation } \\
\text { - La majorité des } \\
\text { importations sont des } \\
\text { produits d'aquaculture }\end{array}$ & $\begin{array}{l}\text { - Efforts } \\
\text { d'harmonisation } \\
\text { réglementaire avec } \\
\text { les partenaires } \\
\text { commerciaux et } \\
\text { certains marchés }\end{array}$ \\
\hline
\end{tabular}




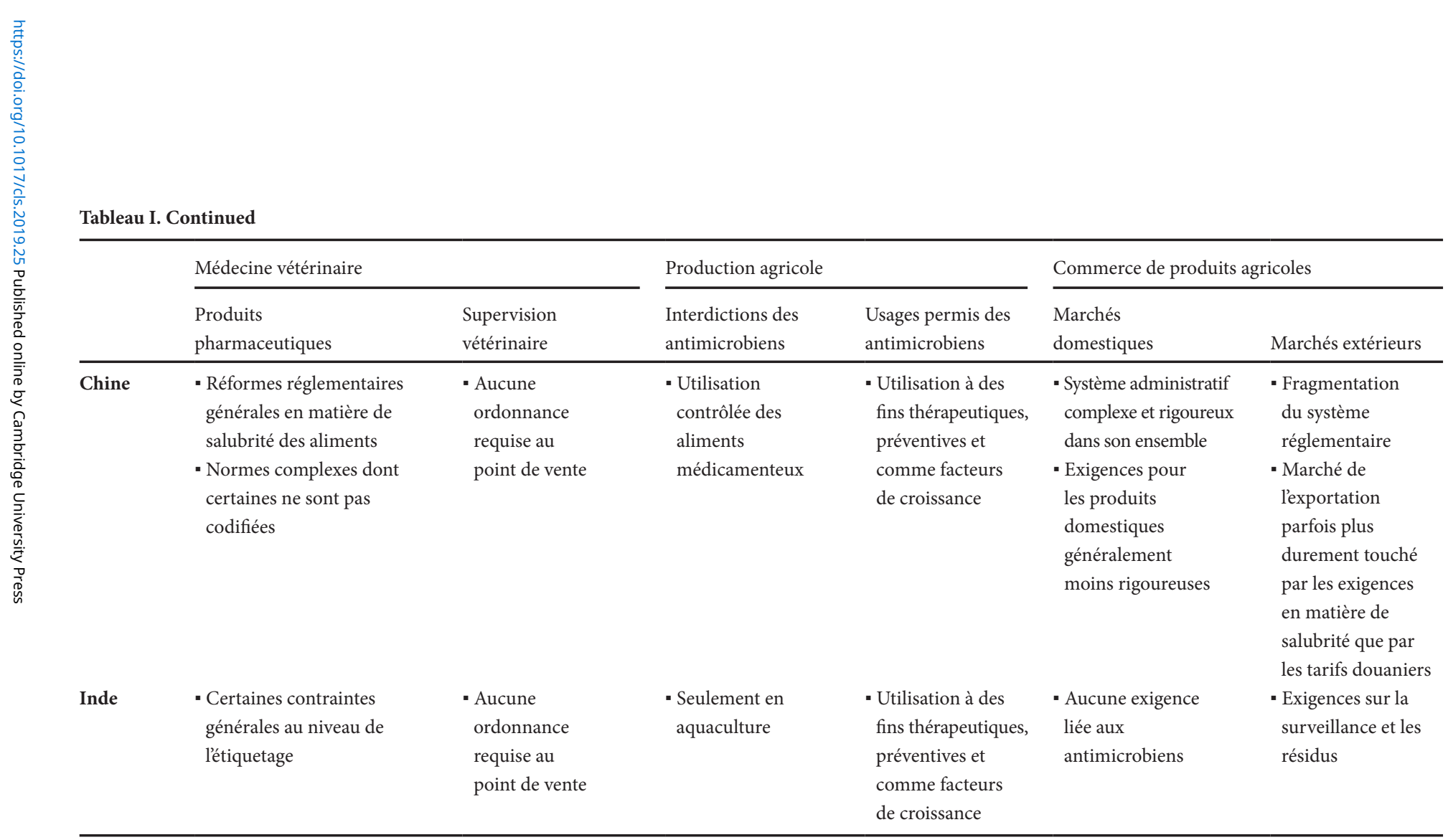

${ }^{*}$ Le « off-label use » se rapporte aux usages autres que ceux indiqués. 
De plus, en raison de failles au niveau de la surveillance, l'étude de l'efficacité des règlements discutés est grandement limitée car elle tient peu compte de l'usage réel, qui peut souvent séloigner des exigences règlementaires.

\section{Réglementation dans dix pays}

\subsection{La France et l'Europe : chefs de file de la réglementation des antimicrobiens}

Le cadre législatif français qui régit l'usage des agents antimicrobiens pour les animaux d'élevage est en grande partie représentatif des mesures qui sont en vigueur dans l'ensemble de l'Union européenne. Ses règlements sur les antimicrobiens ont pour objectif de promouvoir les impératifs de santé publique. Les pays européens ont été les premiers à interdire les facteurs de croissance dans leur ensemble. Ils ont également un cadre réglementaire complet ( de la ferme à la table ») en matière de salubrité des aliments 5 .

Les médicaments vétérinaires sont sujets à des exigences très strictes avant même leur mise en marché. Le processus d'approbation d'un antibiotique à usage vétérinaire tient compte de la qualité, l'efficacité et l'innocuité envers l'utilisateur, le consommateur, l’environnement et l'animal auquel il est destiné. Suite à la mise en marché, un suivi est effectué par l'Agence nationale du médicament vétérinaire à différents égards ${ }^{6}$. La vente est également encadrée : l’obtention des médicaments, soit en pharmacie, soit directement auprès d'un vétérinaire, nécessite une ordonnance ${ }^{7}$.

Au-delà de certains mécanismes communs dans l'ensemble de l'Union européenne, tel le processus d'approbation des médicaments, la France a mis en œuvre ses propres initiatives. Par exemple, le Plan ÉcoAntibio a atteint son objectif ${ }^{8}$ visant à réduire de $25 \%$ l'usage des antibiotiques en médecine vétérinaire en cinq ans ${ }^{9}$. Le cadre réglementaire, cependant, a été critiqué par les professionnels en raison de sa complexité. Cela souligne combien il est important de faciliter l'engagement des professionnels par la formation et la sensibilisation, et de prévoir une communication régulière sur les démarches législatives entreprises ${ }^{10}$.

Le Conseil général de l'alimentation, de l'agriculture et des espaces ruraux (CGAAER) a été chargé d'évaluer le plan Écoantibio qui a pris fin puisque la décision a été prise de renouveler le projet pour cinq ans ${ }^{11}$. Parmi les recommandations, on retrouve la sensibilisation des professionnels en santé animale, ainsi que

5 European Commission, « General Food Law » (20 août 2015)<http://ec.europa.eu/food/safety/ general_food_law/index_en.htm>.

6 Hélène Chardon et Hubert Brugere, Usage des antibiotiques en élevage et filières viandes (Paris : Centre d'Information des Viandes, 2014) <https://www.veterinaire.fr/fileadmin/user_upload/ images/CRO/Languedoc-Roussillon/actualites/AB_Usage_antibiotiques_elevage_CIV_2014_H_ Chardon-H_Brugere_-_copie.pdf $>$, p. 24.

7 Ibid., p. 25-26.

8 Pascale Briand, Catherine Dupuy, Lionel Parle, Le plan Écoantibio 2012-2016 (Ministère de l'agriculture et de l'alimentation) <http://agriculture.gouv.fr/le-plan-ecoantibio-2012-2016>.

9 Chardon et Brugere, p. 26.

10 République française, Ministère de l'agriculture, de l'agroalimentaire et de la forêt, « Rapport $\mathrm{n}^{\mathrm{O}}$ 16041 : Le plan écoantibio 2012-2016, Recommandations pour le plan suivant» (2016), p. 6 <http://www.lafranceagricole.fr/r/Publie/FA/p1/Infographies/Web/2017-04-12/cgaaer_ 16041_2017_rapport.pdf >.

11 Le plan Écoantibio. 
l'éducation du grand public. De plus, du travail reste à faire au niveau des stratégies de surveillance. Entre autres, les indicateurs quantitatifs d'exposition des animaux aux antibiotiques devront être élaborés par espèce et type de production, et être harmonisés avec les méthodes européennes afin de faciliter la comparaison avec les États membres. De plus, si ces indicateurs sont chiffrés, les objectifs devront être proposés par les chaînes de production ${ }^{12}$. Le CGAAER recommande également de prévoir des incitations financières pour les vétérinaires, de la même manière que ce qui est pratiqué en médecine humaine. ${ }^{13}$

En tant que membre de l'Union européenne, la France est sujette à l'interdiction de 2006 des facteurs de croissance chez les animaux d'élevage ${ }^{14}$. L'usage préventif est cependant permis, quoique l'usage préventif de certaines substances antibiotiques d'importance critique soit interdit ${ }^{15}$.

Dans le contexte du commerce, les conditions d'importation sont établies par la Commission européenne qui a compétence en cette matière au nom des vingtcinq états membres, dont la France ${ }^{16}$. En vertu de la directive 96/23/EC, certaines substances sont interdites dans la viande et les produits à base de viande.

En 2014, l'Assemblée nationale de la France a adopté la Loi d'avenir pour l'agriculture, l'alimentation et la forêt, qui a comme objectif une performance plus économique, environnementale et sociale des exploitations agricoles. En février 2016, la loi avait été mise en œuvre à $81 \%{ }^{17}$ La France a également adopté le règlement européen $n^{0} 1760 / 2000^{18}$ et le règlement d'exécution (UE) $n^{0} 1337 / 2013^{19}$ pour rassurer les consommateurs quant à la qualité des produits de viande qu'ils consomment. Ces règlements rendent obligatoire l'étiquetage des pays d'élevage et d'abattage des animaux dont la viande est vendue en France 2021.

Il a également été suggéré que, pour offrir des protections aux producteurs français sur le plan de la concurrence, il devra y avoir une réciprocité entre les mesures imposées par l'Union européenne aux produits de ses pays membres et

12 Ibid.

13 Ibid.

14 Dina Fine Maron, Tyler J. S. Smith et Keeve E. Nachman, « Restrictions on antimicrobial use in food animal production: an international regulatory and economic survey ", Globalization and Health 9 (2013): article 48, p. 4 <http://globalizationandhealth.biomedcentral.com/articles/ 10.1186/1744-8603-9-48>.

15 Journal officiel de la République française, « Décret n ${ }^{\circ}$ 2016-317 » (16 mars 2016). <https://www. legifrance.gouv.fr/affichTexte.do?cidTexte=JORFTEXT000032251629\&categorieLien=id $>$.

16 Commission européenne, Conditions d'importation de la viande fraîche et des produits à la base de viande dans l'UE (European Commission Trade, 2012).

17 République française, "La loi d’avenir pour l'agriculture, l'alimentation et la forêt » (25 mai 2016) <http://www.gouvernement.fr/action/la-loi-d-avenir-pour-l-agriculture-1-alimentationet-la-foret $>$.

18 Direction générale de la concurrence, de la consommation et de la répression de fraudes, «Traçabilité de la viande bovine » (Le portail de l'Économie et des Finances, 2009) <https://www. economie.gouv.fr/node/33546>.

19 EUR-Lex, Règlement d'exécution (UE) n 1337/2013 (13 décembre 2013). <https://eur-lex.europa. eu/legal-content/FR/TXT/PDF/?uri=CELEX:32013R1337\&from=DE $>$.

20 Caroline Evrat Georgel, « Étiquetage de l'origine des viandes : le règlement publié » (23 janvier 2014) <http://idele.fr/presse/publication/idelesolr/recommends/etiquetage-de-lorigine-des-viandesle-reglement-publie.html $>$.

21 Gouvernement de la France, «Crise de lélevage : l'Europe décide de mesures concrètes pour limiter la surproduction » (15 mars 2016) <http://www.gouvernement.fr/argumentaire/crise-de-1elevage-l-europe-decide-de-mesures-concretes-pour-limiter-la-surproduction-4013>. 
celles imposées aux produits importés ${ }^{22}$. Le 30 mai 2018, un projet de loi a été déposé qui porte sur l'équilibre des relations commerciales dans le secteur agricole et une alimentation saine et durable ${ }^{23}$.

\subsection{Le Danemark : avant-gardiste en réglementation des antimicrobiens}

Dès les années 1990, le Danemark a commencé à réglementer l'usage des antimicrobiens, plus d'une décennie avant l'interdiction européenne (et française) des facteurs de croissance en $2006^{24}$. De plus, comme son système réglementaire est appuyé par une surveillance très complète, ses pratiques sont souvent invoquées lors des discussions portant sur la réglementation des agents antimicrobiens. Le Danemark offre un modèle de cadre réglementaire qui tient compte de diverses interdépendances entre les différents acteurs et processus : finalement, malgré l'importance économique de l'élevage au Danemark, des règlements conservateurs ont été adoptés pour combattre la résistance antimicrobienne.

Comme en France, une ordonnance d'un vétérinaire est requise pour acheter des antibiotiques ${ }^{25}$. Mais l'approche du Danemark démontre que l'efficacité de cette mesure peut être grandement améliorée en prévoyant des limites aux profits qu'effectuent les vétérinaires lors de la vente d'antibiotiques. Depuis 1995, les profits effectués par les vétérinaires lors de la vente d'antibiotiques et médicaments vétérinaires sont plafonnés ${ }^{26}$. Autrement dit, les vétérinaires ne peuvent plus approvisionner les fermiers directement en médicaments vétérinaires, sauf si certaines conditions sont remplies. ${ }^{27}$

En 2009, les éleveurs de porcins danois ont volontairement accepté de cesser d'utiliser les céphalosporines; leur consommation a chuté par la suite ${ }^{28}$. En 2015, le taux d'usage d'antimicrobiens dans l'industrie du porc a baissé d'environ $4 \%$ par rapport à l'année précédente, malgré une augmentation de $3 \%$ de la production et $17 \%$ des exportations ${ }^{29}$. Le Danemark démontre donc que les mesures volontaires peuvent connaître un certain succès.

Le Danemark interdit aussi l'utilisation régulière des antimicrobiens à des fins préventives $^{30}$ au-delà des facteurs de croissance. Ceci crée donc une distinction

22

Le plan Écoantibio.

«\#EGalim : l'Assemblée nationale vote le projet de loi en $1^{\text {re }}$ lecture " $<$ http://agriculture.gouv.fr/ egalim-lassemblee-nationale-vote-le-projet-de-loi-agriculture-et-alimentation>

CDDEP Global Antibiotic Resistance Partnership, The State of the World's Antibiotics (2015), à la p. 44. <https://cddep.org/publications/state_worlds_antibiotics_2015/>

Maron, Smith et Nachman, p. 5.

Ibid. p. 6.

Ministry of Environment and Food of Denmark, « Distribution and Use of Veterinary Drugs in Denmark " (2015) <www.foedevarestyrelsen.dk/english/Animal/AnimalHealth/Veterinary_ medicine/Pages/default.aspx $>$.

Yvonne Agersø et Frank M. Aarestrup, "Voluntary ban on cephalosporin use in Danish pig production has effectively reduced extended-spectrum cephalosporinase-producing Escherichia coli in slaughter pigs ", Journal of Antimicrobial Chemotherapy 68, $\mathrm{n}^{\circ} 3$ (2012): 569-572

Birgitte Borck Høg et Leonardo de Knegt, "Chapter 4: Antimicrobial consumption in animals ", dans DANMAP 2015 - Use of antimicrobial agents and occurrence of antimicrobial resistance in bacteria from food animals, food and humans in Denmark (novembre 2016), p. 34.

Carol Cogliani, Herman Goossens et Christina Greko, « Restricting Antimicrobial Use in Food Animals: Lessons from Europe ", Microbe 6, $\mathrm{n}^{\circ} 6$ (2011): 274 p. 278. <https://www.semanticscholar. org/paper/Restricting-Antimicrobial-Use-in-Food-Animals\%3A-from-Cogliani-Goossens/ 6533b1856d5ac6b67728085bfbabe6f0d597abb6> 
claire entre la prévention des maladies et les facteurs de croissance, une distinction qui peut facilement s'estomper lorsque l'usage régulier ou « préventif » est considéré thérapeutique.

Tel qu'indiqué, le système réglementaire danois encadre étroitement les pratiques vétérinaires et l'achat d'agents antimicrobiens. Les mesures ont eu beaucoup de succès suite à leur entrée en vigueur, mais la consommation d'agents antimicrobiens a recommencé à augmenter après quelques années. Ainsi, en 2010, un nouveau système de " cartons jaunes » et de « cartons rouges », qui vise les fermiers, a été adopté. Cette initiative crée un mécanisme de contrôle et de sanctions pour les fermiers dont l'usage d'antimicrobiens dépasse un certain seuil. Elle a pour but de partager une part de cette responsabilité entre les vétérinaires et les fermiers, en responsabilisant ces derniers sur leur usage lors de la production ${ }^{31}$.

Dans le contexte de la production agricole, le Danemark déploie des efforts considérables pour ralentir la résistance aux antimicrobiens depuis les années 1990. Cependant, il est toujours impossible d'assurer que des organismes avec une résistance n'entrent pas au pays par voie du commerce, en raison des importations et des infections liées au transport ${ }^{32}$.

Quant au commerce, le Danemark est parmi les plus grands exportateurs de porc au monde, exportant plus de $90 \%$ du porc qu'il produit. Selon le Conseil danois sur l'agriculture et les aliments, le Danemark s'est mérité une place de premier rang dans les marchés mondiaux en raison de la qualité de ses produits. ${ }^{33}$ Les modes de production durables seraient également un élément clé pour le système de production danois. ${ }^{34}$

Cependant, il faut noter que le marché danois bénéficie de protections; en tant que pays membre de l'Union européenne, le Danemark bénéficie d'avantages par rapport à certains de ses compétiteurs internationaux. Entre autres, les tarifs imposés par l'Union européenne sur les produits provenant de pays non-membres font en sorte que ces produits ne peuvent pas être compétitifs sur le marché européen, malgré le fait que leurs coûts de production sont plus bas. ${ }^{35}$ Au-delà des tarifs, d’autres considérations limitent également l'accès au marché européen pour plusieurs pays non-membres, dont notamment les normes en matière de salubrité des aliments et l'interdiction de la ractopamine ${ }^{36}$. Ces mesures, qui créent certains obstacles au commerce, contribuent donc à la protection du marché danois et aident à assurer sa concurrence sur la scène internationale.

31 Ministry of Environment and Food of Denmark, "The Yellow Card Initiative on Antibiotics " (2015), <https://www.foedevarestyrelsen.dk/english/Animal/AnimalHealth/Pages/The-YellowCard-Initiative-on-Antibiotics.aspx>; Maron, Smith et Nachman, p. 6.

32 Steven J. Hoffman et Trygve Ottersen, "Addressing Antibiotic Resistance Requires Robust International Accountability Mechanisms ", The Journal of Law and Medicine \& Ethics 43, Suppl 3 (2015): 53-64 <https://www.researchgate.net/profile/Trygve_Ottersen/publication/280490424_ Addressing_Antibiotic_Resistance_Requires_Robust_International_Accountability_ Mechanisms/links/55b6255e08ae9289a08aa07a.pdf\#page=38>.

33 Danish Agriculture \& Food Council, " Danish Pig Meat Industry " (Danish Agriculture, 22 août 2018) <http://agricultureandfood.dk/danish-agriculture-and-food/danish-pig-meat-industry>.

34 Ibid.

35 British Meat Processors Association. Pigmeat. <http://britishmeatindustry.org/industry/importsexports/pigmeat/>.

Ibid. 


\subsection{L'Australie : réglementation rigoureuse des médicaments}

L'Australie ne produit pas d'antibiotiques ${ }^{37}$, mais son processus d'approbation d'antibiotiques est parmi les plus conservateurs au monde ${ }^{38}$. Une ordonnance d'un vétérinaire est requise pour se procurer des antibiotiques dans presque tous les $\mathrm{cas}^{39}$, puisque la majorité des antibiotiques vétérinaires figurent parmi la liste de produits à l'annexe 4 du Poisons Standard ${ }^{40}$.

Des contrôles sont effectués à différents égards pour assurer que les médicaments vétérinaires sont utilisés à bon escient, conformément aux règles établies par le gouvernement australien. L'étiquetage, entre autres, restreint l'usage d'agents antimicrobiens dans certaines circonstances ${ }^{41}$. Malgré tout, quelques différences existent quant aux usages hors indication (le "off-label use »), puisque l'utilisation des antibiotiques tombe sous la juridiction des états et territoires australiens ${ }^{42}$. Il s'agit d'une limitation importante du pouvoir fédéral en matière d'adoption des règlements à certains égards, puisque le pouvoir législatif est divisé entre le gouvernement fédéral et les gouvernements des états et des territoires.

Dans le cadre de la production agricole, il n'existe aucune règle interdisant l'usage des facteurs de croissance ${ }^{43}$. L'usage de quelques antimicrobiens particuliers est cependant interdit à cette fin dont, par exemple, les fluoroquinolones ${ }^{44}$. Les vétérinaires peuvent, dans certaines circonstances, prescrire des antibiotiques importants en santé humaine, telles les céphalosporines de troisième génération et les quinolones ${ }^{45}$.

Pour le commerce des produits, puisque le marché européen interdit les facteurs de croissance (permis en Australie), les producteurs australiens qui désirent exporter leurs produits sur le marché européen doivent être certifiés par le European Union Cattle Accreditation Scheme ${ }^{46}$. Cet organisme australien garantit la traçabilité des animaux produits en Australie et assure que les normes européennes sont respectées pour les animaux qui sont destinés à ce marché. ${ }^{47}$

37 T. M. Dyke, « Regulation of veterinary antibiotics in Australia », Communicable Diseases Intelligence 27 (2003) <http://www.health.gov.au/internet/main/publishing.nsf/Content/cda-pubs-cdi-2003cdi27suppl-htm-cdi27supc.htm>.

Australian Commission on Safety and Quality in Health Care, "Australian One Health Antimicrobial Resistance Colloquium Background Paper » (juillet 2013), p. 9 <http://www. safetyandquality.gov.au/wp-content/uploads/2013/07/Briefing-paper-for-One-Health-AMRColloquium-participants-Final-Jul-2013.pdf $>$. CDDEP, State of the World's Antibiotics, p. 46.

"Australian One Health », p. 9.

Laura Gartry, "Study shows Australian livestock industry leading the way in antibiotic resistance " (27 mai 2014) <http://www.abc.net.au/news/2014-05-27/strong-regulation-keeps-antibioticresistance-out-of-livestock/5481172>.

42 «Australian One Health », p. 9.

43 Maron, Smith et Nachman, p. 8.

44 CDDEP « State of the World's Antibiotics », p. 46.

45 "Australian One Health », p. 6; Animals Australia, «Super scary: animal agriculture linked to 'superbug' threat » (22 novembre 2016) <http://www.animalsaustralia.org/features/global-superbugthreat.php $>$.

46 Source : < https://www.business.qld.gov.au/industries/farms-fishing-forestry/agriculture/livestock/ cattle/hormonal-growth-promotants/overseas-market>.

47 Department of Agriculture, European Union Cattle Accreditation Scheme (Australian Government, n.d.) <http://www.agriculture.gov.au/export/controlled-goods/meat/elmer-3/eucas>. 
En dernier lieu, malgré l'existence de programmes de surveillance des pratiques vétérinaires et de la production, l'usage des agents antimicrobiens n'est pas surveillé au-delà du volume de vente ${ }^{48}$.

\subsection{Le Canada : mesures volontaires modérées sur les médicaments}

Le Canada opte davantage pour des mesures volontaires pour la réglementation des agents antimicrobiens. Depuis la publication du cadre d'action Résistance aux antimicrobiens et utilisation de ces derniers au Canada: un cadre d'action fédéral en $2014^{49}$, le Canada s'est engagé à renforcer différents cadres réglementaires et législatifs qui entourent les antimicrobiens.

Depuis quelques années, le gouvernement fédéral travaille de concert avec l'industrie pharmaceutique pour modifier les règlements qui encadrent l'usage d'agents antimicrobiens pour les animaux. Par exemple, de nouvelles règles sur l'étiquetage visent à retirer les affirmations de bienfaits en matière de stimulation de la croissance des animaux d'élevage des étiquettes de médicaments jugés importants sur le plan médical ${ }^{50}$.

Les exigences relatives aux vétérinaires varient d'une province à l'autre ${ }^{51}$. Ces différences réglementaires entre les provinces s'expliquent par la séparation des pouvoirs du système fédéral canadien, une séparation problématique lorsque la réglementation requiert des actions concertées dans plusieurs domaines. Par exemple, en vertu de nouvelles règles fédérales, les acheteurs doivent présenter une ordonnance d'un vétérinaire pour se procurer des aliments médicamenteux contenant des antimicrobiens d'importance en médecine humaine. Quant aux détaillants, ils ne peuvent acheter ou vendre ces produits aux acheteurs qui ont une ordonnance que si les lois de leur province ou territoire le permet ${ }^{52}$.

L'importance des marchés extérieurs pour le commerce du bétail canadien a cependant mené à l'adoption de mesures volontaires telles que l'interdiction de certaines substances lors de la production. Par exemple, en 2013, des producteurs d'Alberta ont accepté de cesser d'utiliser la ractopamine dans la production de porcs, substance interdite dans environ 160 pays. Puisque le Canada exporte plus de viande qu'il n'en importe, cette interdiction a exercé une influence déterminante, suscitant l'adoption de mesures volontaires. ${ }^{53}$ La même année, le gouvernement fédéral

48 Australian Commission on Safety and Quality in Health Care « Antimicrobial resistance: A Report of the Australian One Health Antimicrobial Resistance Colloquium » (18 juillet 2013), p. 12. <https://www.safetyandquality.gov.au/sites/default/files/migrated/Briefing-paper-for-OneHealth-AMR-Colloquium-participants-Final-Jul-2013.pdf $>$.

49 Gouvernement du Canada, "Résistance et recours aux antimicrobiens au Canada : cadre d'action fédéral » (13 novembre 2014). <https://www.canada.ca/fr/sante-publique/services/resistance-auxantibiotiques-antimicrobiens/resistance-et-recours-aux-antimicrobiens-canada-cadre-actionfederal.html>.

50 Health Canada, "Health Canada proposes new measures to address antimicrobial resistance " (17 avril 2015) <http://news.gc.ca/web/article-en.do?nid=965249>.

51 Fine Maron, Smith et Nachman, p. 5.

52 Gouvernement du Canada, « Antimicrobiens importants sur le plan médical et le RNSM » (2019) $<$ http://inspection.gc.ca/animaux/aliments-du-betail/antimicrobiens-chez-les-animaux/aim-etle-rnsm/fra/1521554359053/1521554359444>.

53 Agence canadienne d'inspection des aliments, "Annexe T: Programme canadien de certification des porcs exempts de ractopamine (PCCPER)»<https://www.inspection.gc.ca/aliments/exportationsd-aliments/exigences-particulieres/viandes/pccper/fra/1434119937443/1434120400252>. 
a lancé le Programme canadien de certification des porcs exempts de ractopamine qui encadre différents stades du processus de mise en marché ${ }^{54}$.

Certains suggèrent que le Canada pourrait améliorer sa compétitivité sur la scène internationale, par exemple, en produisant de la viande qui réponde aux demandes grandissantes de la communauté internationale qui requièrent des animaux élevés sans facteurs de croissance ${ }^{55}$. Présentement, pour conserver ses clients européens, le Canada doit maintenir une "double production " pour assurer que les produits destinés au marché européen ne contiennent pas de substances qui y sont interdites, mais qui sont toutefois permises au Canada ${ }^{56}$.

Enfin, l'importation de produits de santé est principalement régie par la Loi sur les aliments et drogues et ses règlements. Cette loi fait la distinction entre l'importation à des fins commerciales et l'importation à des fins d'utilisation personnelle ${ }^{57}$. Cette dernière catégorie crée une faille problématique dans la règlementation en raison du manque de surveillance des produits qui entrent ainsi au pays ${ }^{58}$.

\subsection{Les États-Unis : mesures volontaires initiales sur les médicaments}

Les États-Unis sont des importateurs et exportateurs très importants de bétail sur la scène mondiale. Cependant, les mesures spécifiques aux agents antimicrobiens demeurent, pour la plupart, volontaires.

Des contrôles obligatoires sont effectués avant que la mise en marché d'un médicament soit approuvée ${ }^{59}$, un processus géré par le Food and Drug Administration (FDA). Pour les médicaments destinés aux animaux, le FDA a émis deux documents d'orientation au sujet de l'étiquetage. Le document $n^{0} 209$ n'encourage l'utilisation judicieuse, dans le contexte de l'élevage, des médicaments antimicrobiens importants en médicine humaine qu'à des fins thérapeutiques, et seulement sous la direction d'un vétérinaire ${ }^{60}$. Le document $n^{0} 213$ porte sur

54 Agence canadienne d'inspection des aliments, « Annexe T: Programme canadien de certification des porcs exempts de ractopamine » (2019) <https://www.inspection.gc.ca/aliments/exportationsd-aliments/exigences-particulieres/viandes/pccper/fra/1434119937443/1434120400252>.

55 Cameron Groome, «Industry needs antibiotic alternatives » (30 juillet 2015) < http://www.producer. com/2015/07/industry-needs-antibiotic-alternatives/>.

56 Parlement européen, «Questions parlementaires : Traçabilité des antibiotiques ou des hormones de croissance dans le cadre des importations de viande bovine depuis le Canada dans le cadre du CETA » (2 février 2017) <http://www.europarl.europa.eu/sides/getDoc.do?pubRef=-//EP//TEXT+ $\mathrm{WQ}+\mathrm{P}-2017-000754+0+\mathrm{DOC}+\mathrm{XML}+\mathrm{V} 0 / / \mathrm{FR} \&$ language $=\mathrm{fr}>$.

57 L'utilisation personnelle s'entend de l'importation d'un produit par un particulier pour son utilisation personnelle ou pour une personne ou un animal dont il est responsable à des fins d'utilisation personnelle et non pour la vente. La présente définition ne s’applique pas aux praticiens (médecin, vétérinaire etc.) qui importent des drogues pour traiter leurs patients ou des animaux qui ne leur appartiennent pas. Santé Canada, « Document d'orientation : Les exigences d'importation pour les produits de santé en vertu de la Loi sur les aliments et drogues et ses règlements (GUI-0084)» $\left(1^{\text {er }}\right.$ juin 2010) <http://www.hc-sc.gc.ca/dhp-mps/compli-conform/import-export/gui-0084_biu-uiffra.php>.

58 Ontario Medical Association, «Ontario's Doctors: Antibiotic resistance poses major threat to public health »(20 mars 2013) <https://www.newswire.ca/news-releases/ontarios-doctorsantibiotic-resistance-poses-major-threat-to-public-health-512103981.html>.

59 Animal Health Institute, "Animal Antibiotics: Antibiotic resistance and the role of the animal health industry»<http://www.ahi.org/issues-advocacy/animal-antibiotics/>.

60 US Food \& Drug Administration, "CVM GFI \#209 The Judicious Use of Medically Important Antimicrobial Drugs in Food-Producing Animals » (avril 2012) <https://www.fda.gov/regulatoryinformation/search-fda-guidance-documents/cvm-gfi-209-judicious-use-medicallyimportant-antimicrobial-drugs-food-producing-animals $>$. 
l'étiquetage des nouveaux médicaments antimicrobiens qui ont déjà été approuvés par le FDA ${ }^{61}$. L'avancée la plus importante de ces documents est la recommandation portant sur la supervision vétérinaire.

La directive Veterinary Feed a aussi pour objectif d'augmenter la supervision par des vétérinaires ${ }^{62}$. Cette directive encadre l'usage d'antimicrobiens importants en médecine humaine dans la nourriture des animaux, en exigeant l'autorisation d'un vétérinaire ${ }^{63,64}$. De plus, la nourriture animale qui contient des antimicrobiens d'importance en médecine humaine ne peuvent plus noter les avantages en matière de croissance sur l'étiquette ${ }^{65}$. La directive Veterinary Feed fait cependant l'objet de critiques. Par exemple, plusieurs croient que l'approche volontaire ne prévoit ni des mécanismes pour assurer le respect des mesures, ni des paramètres pour évaluer leur succès. ${ }^{66}$

Dans le contexte de la production agricole, en vertu du document $n^{0} 209$, la distinction entre les agents antimicrobiens dont l'usage devrait être permis et limité est effectuée en fonction de leur importance en médecine humaine ${ }^{67}$. En vertu du document $n^{\circ} 152$, plusieurs antibiotiques, telles les pénicillines et les tétracyclines, sont importants en médecine humaine, et leur étiquetage ne devrait donc pas vanter des bienfaits en matière de stimulation de croissance $^{68}$.

Dans le cadre du commerce, certaines exigences en matière d'exportation sont plus rigoureuses que celles requises pour les produits destinés au marché intérieur. Cette distinction s'explique en partie par des différences entre les régimes règlementaires des États-Unis et de ses partenaires commerciaux. Les États-Unis ont, dans le passé, quand même réussi à négocier des ententes commerciales spéciales avec des pays qui interdiraient autrement leurs produits. Par exemple, suite à l'interdiction européenne concernant l'utilisation des facteurs de croissance, les États-Unis et l'Union européenne ont signé, en 2009, un protocole d'entente qui permettait l'importation de bœuf américain « de haute qualité » en Europe par le biais de contingents tarifaires exemptés des droits de douanes. Cette entente a pris fin le 2 août $2015^{69}$. Un programme de quotas annuels est maintenant en vigueur,

61 Food and Drug Administration, « Guidance for Industry \#213 ( décembre 2013) <http://www. fda.gov/downloads/AnimalVeterinary/GuidanceComplianceEnforcement/GuidanceforIndustry/ UCM299624.pdf>.

62 US Department of Health and Human Services, "Veterinary Feed Directive for Animal Producers " (22 décembre 2016) <https://www.fda.gov/AnimalVeterinary/SafetyHealth/AnimalFeedSafety SystemAFSS/ucm534246.htm>.

63 Animal Health Institute, «FDA Guidance 209, 213 and VFD Educational Material ».

64 US Department of Health and Human Services.

65 Ibid.

66 Federal Register, Veterinary Feed Directive, « Comment 43 » (2015) <https://www.federalregister. gov/documents/2015/06/03/2015-13393/veterinary-feed-directive $>$.

67 Ibid.

68 Food and Drug Administration, p. 5.

69 Carol Guthrie, «U.S. Trade Representative Froman, Secretary of Agriculture Vilsack Announce Continued EU Market Access for American Producers of High-Quality Beef » $\left(1^{\text {er }}\right.$ août 2013) <http://www.usda.gov/wps/portal/usda/usdahome?contentid=2013/08/0152.xml\& contentidonly=true $>$. 
limitant la quantité de viande de « haute qualité » qui peut être exportée vers l'Union européenne ${ }^{70}$.

\subsection{La Russie : politique, embargos et impact sur le commerce international}

Ces dernières années, des considérations de politique internationale ont fait que le marché de la viande a beaucoup changé en Russie, en partie en raison des changements apportés aux politiques gouvernant le commerce des produits d'élevage. De même, il n'existe que peu de restrictions quant aux antimicrobiens et aux usages permis. Le législateur a cependant apporté plusieurs modifications au cadre législatif gouvernant la mise en marché des produits pharmaceutiques vétérinaires au cours des dernières années. L'accent semble être mis sur le produit final et le commerce.

Ces dernières années, la Russie a renforcé le cadre législatif dans lequel s'inscrivent la surveillance, l'approbation, l'enregistrement et la circulation des produits pharmaceutiques vétérinaires $^{71}$. Par exemple, en 2015, la loi 241-FZ, qui régit la circulation des produits médicaux, a été modifiée pour inclure une disposition sur le processus d'enregistrement des produits pharmaceutiques vétérinaires ${ }^{72}$. Ces changements aideront à clarifier certains aspects administratifs, tel l'enregistrement des produits. Par ailleurs, il n'est pas nécessaire d'obtenir une ordonnance d'un vétérinaire pour obtenir des antibiotiques pour des animaux d'élevage ${ }^{73}$.

Quant au commerce, létude de l'approche russe laisse entrevoir l'importance des considérations politiques sur les marchés mondiaux. En 2014, la Russie a interdit l'importation de porc européen, citant des inquiétudes par rapport à la peste porcine africaine. Plus tard cette même année, la Russie a créé un embargo sur plusieurs produits de porc et de bœuf provenant entre autres de l'Australie, du Canada, des États-Unis et de l'Union européenne. Cette mesure était une réponse aux sanctions économiques imposées à la Russie en raison de son conflit avec l'Ukraine. En 2017, la Russie a augmenté la portée de l'embargo, interdisant l'importation de produits animaliers additionnels ${ }^{74}$.

70 USDA Foreign Agricultural Service. GAIN Report \#E18036 (Global Agricultural Information Network, 2018) <https://gain.fas.usda.gov/Recent\%20GAIN\%20Publications/Comparison\%20 of\%20EU\%20Tariff\%20Rate\%20Quotas\%20for\%20High\%20Quality\%20Bovine\%20Meat_ Brussels\%20USEU_EU-28_5-31-2018.pdf>.

71 Alexander Panin, "Implementation of the Russian Pharmacovigilance regulation " <https:// slideplayer.com/slide/11657326/> <https://www.google.ca/url?sa=t\&rct=j\&q=\&esrc=s\&source= web\&cd=3\&cad=rja\&uact=8\&ved=0ahUKEwjFk-m48rPMAhUlnoMKHb14AgQQFggyMAI\& url=http\%3A\%2F\%2Fvichsec.org\%2Foutreach-forum\%2Findex.php\%3Foption\%3Dcom_ attachments $\% 26$ view\%3Dattachments $\% 26$ task $\% 3$ Dattachment $\% 26 \mathrm{id} \% 3 \mathrm{D} 941 \& u s g=$ AFQjCNHBP1hpC03cXuHjJmPTykLUo4-oUg>.

72 Vsevolod Tyupa, "Russian Law on Circulation of Medicines - Significant amendments to come into force on 1 July 2015 » (15 décembre 2014) <http://www.lexology.com/library/detail.aspx?g= 710ebbe9-cca5-40eb-ba8b-e429d53b6c8f>.

73 Hilde Kruse, "Consumption of antimicrobials in food animals outside EU/EEA » (4-5 février 2014) à la diapositive 12 <http://www.ema.europa.eu/docs/en_GB/document_library/Presentation/ 2014/03/WC500162571.pdf $>$.

74 U.S. Meat Export Federation Staff. Russia expands import embargo: What does it mean for pork trade? National Hog Farmer (2017) <https://www.nationalhogfarmer.com/marketing/russiaexpands-import-embargo-what-does-it-mean-pork-trade>. 
L'Union européenne a déposé une plainte auprès de l'Organisme mondial du commerce (OMC) alléguant que la première interdiction susmentionnée de la Russie, datant de 2014, était contraire à l'Accord sur l'application des mesures sanitaires et phytosanitaires. L'OMC a effectivement trouvé que l'interdiction nétait pas conforme et a émis des recommandations que la Russie a acceptées ${ }^{75}$. Cependant, même si cette interdiction est levée, les embargos subséquents font en sorte que peu de choses changeront sur le plan du commerce entre la Russie et l'Union européenne ${ }^{76}$.

\subsection{Le Japon : salubrité des aliments et des importations}

Puisque le marché du bétail au Japon est principalement un marché d'importation, le cadre réglementaire est axé sur l'importation des produits et la salubrité des aliments.

Des exigences particulières s'appliquent à l'étiquetage de certains produits antimicrobiens. Par exemple, les fluoroquinolones et les céphalosporines de troisième génération ne peuvent pas être utilisés comme médicaments de premier recours et leur étiquetage doit contenir une indication à cet effet. Le processus d'approbation de ces médicaments est également strict. Les normes de gestion du risque des antimicrobiens, fondées sur les lignes directrices de l'OIE, du Codex et du FDA, ont été adoptées pour réduire les effets négatifs sur la santé humaine ${ }^{77}$.

$\mathrm{Au}$ Japon, il est nécessaire de présenter une ordonnance d'un vétérinaire pour se procurer des antimicrobiens pour des animaux d'élevage ${ }^{78}$. Si un vétérinaire le juge nécessaire, un antibiotique peut être utilisé hors indication ( off-label use ») ${ }^{79}$.

Dans le contexte de la production agricole, il est permis d'utiliser des agents antimicrobiens pour stimuler la croissance des animaux ${ }^{80}$. L'usage d'antimicrobiens comme additifs alimentaires est aussi permis si ces antimicrobiens figurent sur une liste exhaustive ${ }^{81}$.

$\mathrm{Au}$ Japon, la réglementation du marché est particulièrement importante, puisque plus de $60 \%$ de la nourriture qui y est consommée provient des marchés extérieurs $^{82}$. De plus, contrairement à la distribution des rôles dans la plupart des marchés, il incombe aux importateurs japonais - et non au gouvernement d’assurer que des médicaments vétérinaires interdits n’ont pas été utilisés lors de la

75 Organisme mondial du commerce, « Fédération de Russie - Mesures visant l’importation de porcins vivants, de viande de porc et d'autres produits du porc en provenance de l'Union européenne » (21 mars 2017) <https://www.wto.org/french/tratop_f/dispu_f/cases_f/ds475_f.htm>.

76 U.S. Meat Export Federation Staff.

77 National Veterinary Assay Laboratory, Ministry of Agriculture, Forestry and Fisheries, « A Report on the Japanese Veterinary Antimicrobial Resistance Monitoring System 2008-2011» (2013), p. 19 <http://www.maff.go.jp/nval/tyosa_kenkyu/taiseiki/pdf/jvarm2008_2011.pdf>. Fine Maron, Smith et Nachman, p. 5.

79 Luca Guardabassi, Lars B. Jensen et Hilde Kruse, "Guide to Antimicrobial Use in Animals » (extrait), p. 73.

80 Fine Maron, Smith et Nachman, p. 5.

81 Kristi O Smedley, « Comparison of Regulatory Management of Authorized Ingredients, Approval Processes, and Risk-Assessment Procedures for Feed Ingredients » (juillet 2013), p. 23 <http:// www.ifif.org/uploadImage/2013/7/19/272ee931adc6dacaadd762c71f8110a31374224070.pdf >.

82 Edward I. Broughton et Damian G. Walker, " Policies and practices for aquaculture food safety in China », Food Policy 35, n 5 (2010): 474. 
création des produits qui entrent au pays, et que les limites législatives japonaises pour les résidus et les additifs alimentaires sont respectées ${ }^{83}$. Le contrôle en matière de salubrité des aliments est particulièrement rigoureux ${ }^{84}$.

\subsection{Le Brésil : production, exportation et harmonisation}

Le Brésil est un des plus grands producteurs de viande au monde ${ }^{85}$. En 2010, ce pays figurait parmi les cinq plus grands consommateurs d'agents antimicrobiens pour les animaux d'élevage au monde ${ }^{86}$. Bien que son système réglementaire ne prévoie que peu d'interdictions quant à l'utilisation des agents antimicrobiens, son système de production et de contrôle tient compte des exigences de ses partenaires commerciaux. Le Brésil produit également des aliments médicamenteux pour les animaux d'élevage, dont certains contiennent des antibiotiques et ont l'objectif de stimuler la croissance des animaux. Malgré le fait que plusieurs juridictions interdisent ces produits, ce marché poursuit son expansion, en raison de la demande d'approvisionnement en viande, ainsi que de la taille des marchés qui utilisent ou permettent toujours l'utilisation de ces substances.

L'industrie pharmaceutique du Brésil est florissante ${ }^{87}$. Environ $40 \%$ des médicaments consommés sur son territoire sont des antibiotiques ${ }^{88}$. En 2010, à la suite d'une série de décès liés à la résistance antimicrobienne, l'agence nationale de surveillance de la santé (Agência Nacional de Vigilância Sanitária) a émis des procédures à suivre lors de l’ordonnance de médicaments antimicrobiens ${ }^{89}$.

Certains médicaments vétérinaires sont régis par une directive émise en $2012^{90}$. Celle-ci établit un éventail de règles, dont certaines sont rigoureuses. Par exemple, le règlement impose des limites à la longueur des traitements qui peuvent être prévus par une seule ordonnance ${ }^{91}$. Depuis 2010, une loi est en vigueur qui exige qu'une ordonnance d'un vétérinaire soit présentée pour acheter des antibiotiques $^{92}$.

Au Brésil, le marché des additifs alimentaires est énorme et la catégorie des aliments médicamenteux contenant des antibiotiques continuera à prendre de

83 Ibid.

84 Ibid., p. 475.

85 FAOSTAT, «Production quantities by country » <http://www.fao.org/faostat/en/\#data/QAL $>$.

86 Thomas P. Van Boeckel, «Global trends in antimicrobial use in food animals », Proceedings of the National Academy of Sciences 112, $\mathrm{n}^{\circ} 18$ (2015): 5650.

87 IMS Health, "Pharmerging markets » (2013) <https://www.imshealth.com/files/web/Global/ Services/Services\%20TL/IMS_Pharmerging_WP.pdf $>$.

88 Yared Santa-Ana-Tellez, «Impact of Over-the-Counter Restrictions on Antibiotic Consumption in Brazil and Mexico ", PLOS One 8, n 10 (2013): e75550, p. 2, doi: 10.1371/journal.pone.0075550. CRMV, « Prescrição de Receituário para Antimicrobianos » (8 mai 2013) <http://www.crmv-mt. org.br/index.php/artigos?id=129>.

90 Ministério da Agricultura, Pecuária e Abastecimento, «Produtos Veterinários » (2014)<http://www. agricultura.gov.br/assuntos/insumos-agropecuarios/insumos-pecuarios/produtos-veterinarios/ produtos-veterinarios $>$.

91 Instrução Normativa 25/2012, Ministério da Agricultura, Pecuária e Abastecimento <http:// sistemasweb.agricultura.gov.br/sislegis/action/detalhaAto.do?method=visualizarAtoPortalMapa\& chave $=573826556>$.

92 Global Legal Monitor, «Brazil: Antibiotics to be sold with prescription only » (5 novembre 2010) $<$ http://www.loc.gov/law/foreign-news/article/brazil-antibiotics-to-be-sold-with-prescriptiononly/>. 
l'ampleur pendant les années à venir ${ }^{93}$. L'utilisation d'antibiotiques chez les animaux à des fins thérapeutiques et de prévention, ainsi que comme facteurs de croissance, est permise ${ }^{94}$.

En matière d'élevage d'animaux, le marché brésilien est axé sur la production et l'exportation. Le marché de l'importation est considérablement plus petit et porte principalement sur les produits de l'aquaculture ${ }^{95}$. Puisque l'exportation des produits est d'une grande importance, l'harmonisation internationale est une considération réglementaire soutenue. Par exemple, depuis 1998, le Brésil utilise un système d'analyse des risques et de maîtrise des points critiques en matière de salubrité des aliments, le HACCP. Ce système établit les procédures et les normes requises par plusieurs des marchés vers lesquels le Brésil exporte des produits de viande $^{96}$. Pour ce qui est des exportations de bœuf vers le marché européen, l'usage d'antibiotiques est contrôlé par les usines de transformation de la viande ${ }^{97}$. Cependant, un scandale récent illustre que ce genre de mesure n'est pas suffisamment rigoureux. En 2017, des importants producteurs de viande du pays ont fait l'objet d'allégations de corruption, selon lesquelles ces compagnies auraient offert des pots-de-vin à des inspecteurs pour faire certifier de la viande avariée. ${ }^{98}$

Enfin, le Brésil est un pays membre de Mercosur, un regroupement de pays d'Amérique latine. En 2000, Mercosur a adopté un règlement qui impose des limites aux doses journalières d'antibiotiques administrées aux animaux, ainsi qu'aux résidus de médicaments vétérinaires dans les aliments d’origine animale ${ }^{99}$. Un des objectifs visés par ce règlement est l'harmonisation des règlements techniques pour éliminer les obstacles liés aux divergences entre les approches des différents pays ${ }^{100}$.

\subsection{La Chine : commerce et économie}

En Chine, le marché du bétail est en pleine effervescence. De plus, le contexte réglementaire dans lequel s'inscrit la résistance aux antimicrobiens pour les

93 PRNewswire, « Brazil Animal Feed Additives Market is Expected to Reach USD 1,941.6 Million in 2018: Transparency Market Research » $\left(1^{\mathrm{er}}\right.$ octobre 2012) <http://www.prnewswire.com/newsreleases/brazil-animal-feed-additives-market-is-expected-to-reach-usd-19416-million-in-2018transparency-market-research-172059941.html>.

94 Ministério da Agricultura.

95 OEC, «Imports » (2014) <http://atlas.media.mit.edu <http://atlas.media.mit.edu/en/profile/ country/bra/\#Imports $>$.

96 Patrick Bruha, « HACCP in Brazil » $(20$ novembre 2014) <http://thebrazilbusiness.com/article/ haccp-in-brazil >.

97 Danillo Domingues Millen, Rodrigo Dias Lauritano Pacheco, Paula M. Meyer, Paulo H. Mazza Rodrigueset Mario De Beni Arrigoni, , Current outlook and future perspectives of beef production in Brazil " Animal Frontiers 1, n 2 (2011): 46.

98 Aljazeera, "Brazil's rotten meat scandal prompts major import bans » (2017) <http://www.aljazeera. com/news/2017/03/brazil-rotten-meat-scandal-prompts-major-import-bans-170320185259468. html>.

99 Néstor Falcón, Carmelo Ortega, Silvana Gorniak, Luis Carlos Villamil, Cristina Ríos et Mari Carmen Simón, "El problema de la resistencia a antibióticos en salud pública », Una Salud. Revista Sapuvet de Salud Pública 1 (2009): 85 <http://revistas.lasalle.edu.co/index.php/us/article/ viewFile/235/176>.

100 Reglamento Técnico Mercosur Metodologías Analíticas, Ingesta Diaria Admisible y Límites Máximos de Residuos Para Medicamentos Veterinarios en Alimentos de Origen Animal <http://www.inmetro. gov.br/barreirastecnicas/PDF/GMC_RES_2000-054.pdf>. 
animaux d’élevage subit déjà des changements importants. Puisque la Chine est au premier rang des marchés de l'exportation et de l'importation à l'échelle mondiale, plusieurs réalités se font concurrence. À l'heure actuelle, le gouvernement semble davantage préoccupé par des considérations économiques et sociales, telle la demande de viande tant par les marchés domestiques qu’extérieurs. Conséquemment, des mesures de santé publique qui viseraient à limiter l'usage des facteurs de croissance sont soupesées à la lumière d'autres considérations.

Les réformes en cours en matière de réglementation visent surtout à aborder des problèmes es et fondamentaux, comme les inquiétudes qui ont été notées par rapport à la salubrité des aliments chinois. ${ }^{101}$ Par exemple, pour ce qui est des agents antimicrobiens, il n'est pas nécessaire d'obtenir une ordonnance d'un vétérinaire ${ }^{102}$. Cependant, les objectifs du plan d'action national sur la résistance aux antimicrobiens publié par le gouvernement chinois en 2016 incluent des améliorations au niveau de la surveillance, dont de nouvelles exigences en matière d'ordonnances pour $2020^{103}$.

Dans le contexte de la production, la Chine n'a pas adopté de règlements spécifiques sur l'utilisation d'antimicrobiens pour stimuler la croissance des animaux d'élevage $^{104}$. À quelques exceptions près, celle-ci est toujours permise, ainsi que l'usage préventif et, bien évidemment, l'usage thérapeutique ${ }^{105}$.

Le système réglementaire chinois en matière de salubrité des aliments est davantage axé sur les produits et le commerce que la production ${ }^{106}$. Le système administratif qui régit les importations est très complexe ${ }^{107}$; le contrôle, de façon générale, est rigoureux.

Pour les produits destinés à des marchés extérieurs, les exigences varient. Mais, dans leur ensemble, les exigences en matière de salubrité des aliments semblent plus légères pour les produits destinés aux marchés intérieurs, une tendance commune parmi les marchés en voie de développement ${ }^{108}$. Cette division des normes pour l'importation et l'exportation s'explique entre autres par des faiblesses au niveau de l'infrastructure. Les exportateurs favorisent le marché de l'exportation puisque les marges de profits au sein des marchés domestiques sont beaucoup moins attrayantes. Quant au gouvernement, l'imposition des produits d'exportation peut lui rapporter des sommes importantes; il gagne donc à encourager le marché de l'exportation ${ }^{109}$.

\footnotetext{
101 Broughton et Walker, note 100, p. 477.

102 Maron, Smith et Nachman, note 11, p. 5

103 Kathleen McLaughlin, "China tackles antimicrobial resistance " (31 août 2016) <http://www. sciencemag.org/news/2016/08/china-tackles-antimicrobial-resistance $>$.

104 Maron, Smith et Nachman, p. 5.

105 USDA Foreign Agriculture Service «China - People's Republic of: List of Veterinary Drugs Banned for Use for Food Animals » (2011), p. 4 <http://gain.fas.usda.gov/Recent\%20GAIN\%20 Publications/List\%20 of\%20Veterinary\%20Drugs\%20Banned $\% 20$ for $\% 20$ Use $\% 20$ for $\% 20$ Food\%20Animals_Beijing_China\%20-\%20Peoples\%20Republic\%20of_3-11-2011.pdf>.

107 Broughton et Walker, p. 474.

John Balzano, « Lingering Food Safety Regulatory Issues for China in 2016 » (10 janvier 2016) <http://www.forbes.com/sites/johnbalzano/2016/01/10/lingering-food-safety-regulatoryissues-for-china-in-2016/\#195accd35f5a>.

Ibid.
} 


\subsection{L'Inde : vide réglementaire sur les antimicrobiens}

Au total, l'Inde produit environ 6,3 millions de tonnes de viande chaque année ${ }^{110}$. En 2015, environ 2 millions de tonnes métriques de bœuf et de veau ont été exportées vers des marchés extérieurs, situant l'Inde au premier rang mondial des exportations pour ces produits ${ }^{111}$. La présence de pathogènes résistants aux antimicrobiens dans les produits animaliers indiens peut donc avoir des répercussions importantes ailleurs dans le monde.

L'Inde utilise de grandes quantités d'antimicrobiens chez les animaux délevage mais n'a pas encore adopté de règlements concernant leur utilisation ${ }^{112}$. Bien qu'il contienne quelques interdictions générales, le cadre règlementaire entourant les médicaments vétérinaires et leur vente est également silencieux sur ce sujet. Les lois pertinentes en l'espèce sont principalement axées sur l'aquaculture et les produits destinés aux marchés extérieurs ${ }^{113}$.

Depuis 2013, il est nécessaire d'indiquer le délai d'attente sur les étiquettes d'antibiotiques destinés aux animaux ${ }^{114}$. À quelques exceptions près ${ }^{115}$, une ordonnance d'un vétérinaire n'est nécessaire ni pour les antibiotiques, ni pour les produits d'alimentation contenant des antibiotiques ${ }^{116}$.

En 2014, le gouvernement fédéral a demandé aux états d’encourager l'utilisation judicieuse des antibiotiques de la part des vétérinaires et de décourager l'utilisation d’antibiotiques dans la nourriture des animaux délevage. Cependant, aucun mécanisme de contrôle n'est prévu pour surveiller la mise en œuvre ${ }^{117}$. De plus, bien que le gouvernement fédéral puisse adopter des lois, la mise en œuvre s'effectue au niveau des états ${ }^{118}$.

En fait, toute directive qui mise sur les vétérinaires pour contrôler l'accès au antimicrobiens sera confrontée à des problèmes pratiques considérables. Par exemple, le manque de vétérinaires est un problème grave en Inde ${ }^{119}$. Des mesures réglementaires qui ont connu du succès dans d'autres pays, comme l'exigence d'une ordonnance de vétérinaire, auront alors un effet bien différent dans le contexte indien.

Enfin, l'utilisation d'antimicrobiens dans le domaine agricole est gérée par les différents gouvernements des différents états indiens. Les antimicrobiens sont utilisés à des fins thérapeutiques, pour prévenir les maladies et pour stimuler la

110 CDDEP, « Antibiotic Use and Resistance in Food Animals » (2016), p. 12 <http://www.cddep.org/ sites/default/files/india_abx_report.pdf $>$.

111 United States Department of Agriculture, «Livestock and Poultry: World Markets and Trade " (avril 2016), $6<$ https://timedotcom.files.wordpress.com/2015/04/livestock_poultry.pdf >.

112 Ramanan Laxminarayan, Ranjit Roy Chaudhury, " Antibiotic Resistance in India: Drivers and Opportunities for Action ", PLOS Med 13, no 3 (2016) e1001974<http://journals.plos.org/ plosmedicine/article?id=10.1371/journal.pmed.1001974>.

113 CDDEP, « Antibiotic Use », p. 14.

114 Centre for Science and Environment, « Regulatory Landscape in India » (2014), p. $2<$ http://www. cseindia.org/userfiles/factsheets/factsheet\%204.pdf > .

115 CDDEP, «Antibiotic Use », p. 14.

116 Ibid.

117 Centre for Science and Environment, p. 3.

118 Menaka Rao, "India has a massive antibiotic resistance problem, and it's up to the states to solve it" (27 septembre 2016) <https://scroll.in/pulse/817377/india-has-a-massive-antibiotic-resistance- 
croissance des animaux d'élevage. Souvent, ils sont ajoutés à la nourriture des animaux $^{120}$. En 2007, une recommandation avait été émise de cesser d'utiliser systématiquement des antibiotiques pour la volaille mais il s'agissait cependant d'une mesure volontaire $^{121}$. Seuls les produits provenant de l'aquaculture sont sujets à des interdictions et limites ${ }^{122}$.

\section{Discussion}

Tel qu'illustré dans la section précédente, l'usage d'agents antimicrobiens s'inscrit dans un cadre réglementaire complexe dans lequel les activités de plusieurs acteurs sont régies. À l'heure actuelle, les approches des plus grands exportateurs et importateurs ne sont pas harmonisées en matière de réglementation de la médecine vétérinaire, de la production agricole et du commerce des produits agricoles. Létude et la comparaison des différentes approches adoptées permettent de déceler certaines interdépendances clés qui, si elles sont bien encadrées, améliorent l'efficacité des règlements adoptés. Elles permettent également de déceler les principaux obstacles ainsi que les incitations qui pourraient être déterminantes pour les industries concernées.

\subsection{Observations générales}

Lexamen des approches réglementaires des dix pays identifiés révèle certaines tendances. Les pays industrialisés dont les marchés sont bien établis tendent à adopter des règlements qui agissent plus tôt dans le processus agricole : ils encadrent davantage l'approbation et la vente des médicaments vétérinaires ainsi que leur utilisation lors de la production. Les considérations de santé publique se trouvent au cœur de la réglementation. L'exemple des pays de l'Union européenne démontre que cette approche peut permettre d'établir des normes minimales communes, qui peuvent être renforcées par des exigences plus rigoureuses dans les juridictions qui ont la capacité et la volonté nécessaires.

Les pays en voie de développement dont les marchés sont en pleine évolution, comme la Chine et le Brésil, sont davantage gouvernés par des considérations d’ordre pratique et économique. Par conséquent, ils optent plutôt pour des mécanismes réglementaires régissant le commerce des produits agricoles, une approche axée sur les produits finis. Cette approche est parfois caractérisée par une fragmentation réglementaire importante, ainsi que des lacunes générales au niveau de l'infrastructure qui limitent la capacité de surveillance et de contrôle.

Quelques pays semblent particulièrement tiraillés entre les considérations d’ordre économique et de santé publique. Le Canada et les États-Unis, par exemple, sont des pays industrialisés qui possèdent des marchés bien établis. Ils ont démontré une certaine volonté d'agir, surtout en médecine vétérinaire, mais font surtout appel à des mesures volontaires.

\footnotetext{
120 Centre for Science and Environment, p. 1.

121 Ibid., p. 3.

122 Dave Love, "India develops sound policy on antibiotic use in aquaculture and food animals " (2 mai 2011) <http://www.livablefutureblog.com/2011/05/india-develops-sound-policy-onantibiotic-use-in-aquaculture-and-food-animals $>$.
} 
Les points saillants de ces différentes approches réglementaires sont discutés à la lumière de facteurs contextuels qui peuvent exercer une influence déterminante sur les politiques.

\subsection{Médecine vétérinaire}

\subsubsection{Grande efficacité de cette sphère de réglementation}

La réglementation en médecine vétérinaire peut être très efficace puisqu’elle permet de régler le problème à la source en contrôlant l'accès à certains produits, soit en réglementant leur mise en marché (par exemple, les exigences d'étiquetage), soit en réglementant leur achat (par exemple, les exigences d'ordonnances de vétérinaires). L'Australie est un très bon exemple de l'efficacité de telles mesures. Bien que ce pays n'ait pas de règlements qui établissent des interdictions systémiques au niveau de la production et de lélevage, il bénéficie de règlements très stricts au niveau de la médecine vétérinaire et des produits pharmaceutiques ${ }^{123}$.

Cette approche pourrait être attrayante pour des pays qui veulent agir pour contrôler la résistance aux antimicrobiens sur leur territoire, même si les interdictions législatives sont impossibles dans l'immédiat. Pour les pays qui ont la capacité d'agir mais qui hésitent à prendre position sur les antimicrobiens en particulier, il semblerait que des règlements d’application plus générale puissent contribuer du moins en partie à la lutte contre la résistance antimicrobienne. Plusieurs des pays étudiés qui avaient peu agi en matière de réglementation des antimicrobiens avaient agi sur ce plan dans les dernières années.

\subsubsection{Limites aux profits obtenus par les vétérinaires}

La réglementation danoise démontre que l'exigence d'une ordonnance d'un vétérinaire ne créera pas, à elle seule, une baisse considérable de la consommation d’agents antimicrobiens. La combinaison de cette mesure avec la plafonnement des profits effectués par les vétérinaires est bien plus efficace.http://www.safetyandquality. gov.au/wp-content/uploads/2013/07/Briefing-paper-for-One-Health-AMRColloquium-participants-Final-Jul-2013.pdf Sans une telle limite, la possibilité de profits peut créer une incitation qui minerait l’efficacité du système règlementaire.

\subsubsection{Rôle des vétérinaires}

Les changements réglementaires dans le domaine de la médecine vétérinaire indiquent que la tâche de combattre la résistance antimicrobienne est, de plus en plus, transférée des fermiers aux vétérinaires ${ }^{124}$. Mais la profession de la médecine vétérinaire entretient des liens avec l'industrie pharmaceutique. Lapparition de conflit d'intérêts survient lorsque les vétérinaires peuvent, à la fois, prescrire et distribuer les médicaments ${ }^{125}$. Les désavantages de ce transfert de responsabilité, réels ou perçus, peuvent être atténués grâce à d’autres règlements qui soulignent la transparence.

\footnotetext{
123 « Australian One Health».

124 Jim Fairles, "The Veterinarian's Role in Antimicrobial Stewardship », Canadian Veterinary Journal 54, n 3 (2013): 207 <http://www.ncbi.nlm.nih.gov/pmc/articles/PMC3573624/>. 
Ladoption de solutions autres que l'interdiction des antimicrobiens pourrait également aider à apaiser certaines inquiétudes des vétérinaires en leur permettant de réduire la consommation d'antibiotiques sans compromettre la santé des $\operatorname{animaux}^{126}$. La médecine vétérinaire est effectivement confrontée à un défi de taille : équilibrer la santé des animaux et la préservation de l'utilité des antimicrobiens $^{127}$.

\subsection{Production agricole}

\subsubsection{Niveau d'industrialisation}

Les conséquences économiques de l'adoption de règlements plus rigoureux pourraient être beaucoup plus ressenties dans les marchés moins industrialisés, ce qui peut influencer les décideurs qui envisagent une réglementation antimicrobienne. Ceux-ci utilisent souvent des antibiotiques pour pallier des lacunes du système de production, notamment au niveau de la salubrité ${ }^{128}$. Dans grand nombre de pays en voie de développement, du moins à court terme, les effets négatifs liés à l'adoption de règles plus rigoureuses l'emportent tout simplement sur les effets positifs potentiels $^{129}$.

Dans plusieurs pays en voie de développement, les infections bactériennes sont très communes et sont traitées avec des antimicrobiens ${ }^{130}$. Dans ces contextes, l'amélioration des pratiques d'hygiène pourrait grandement réduire la consommation d'antimicrobiens ${ }^{131}$. Des réformes réglementaires sur la salubrité des aliments et les pratiques de production pourraient donc être une option intéressante de caractère durable ${ }^{132}$.

\subsubsection{Facteurs de croissance et prévention}

Au niveau de la production agricole, l'usage d'antimicrobiens à des fins de traitement est universellement accepté et est considéré comme bénéfique pour la santé des animaux d'élevage et à la santé publique. Les facteurs de croissance font lobjet de bien des débats. Les antimicrobiens utilisés à des fins de prévention compliquent

126 College of Veterinarians of Ontario, « Use of Antibiotics in Food-Producing Animals: Facilitated discussions with Ontario Veterinarians involved with food-producing animal practice » (2015) $<$ https://cvo.org/CVO/media/College-of-Veterinarians-of-Ontario/Resources\%20and\%20 Publications/Reports/GF2DiscussionSummary.pdf $>$.

127 Jean Gauvin, « The CVMA and Prudent Use of Antimicrobials », Canadian Veterinary Journal 55, n 9 (2014): 809 <http://www.ncbi.nlm.nih.gov/pmc/articles/PMC4137919/>.

128 Ramanan Laxminarayan, Thomas Van Boeckel et Aude Teillant, "The Economic Costs of Withdrawing Antimicrobial Growth Promoters from the Livestock Sector ", OECD iLibrary 78 (2015): 5 <http://www.oecd-ilibrary.org/docserver/download/5js64kst5wvl.pdf?expires= 1462842342\&id=id\&accname=guest\&checksum=1D60E7B51DA74E1AAB9FE98FD3009FFA > .

129 Honda Keiichiro, Tsunehiro Otsuki et John S Wilson, « Food Safety Standards and International Trade: The impact on developing countries' export performance ", Food Safety, Market Organization, Trade and Development 151 (2015) (résumé) <http://link.springer.com/chapter/ 10.1007/978-3-319-15227-1_8>.

130 Amornthep Archawakulathep et al, « Perspectives on Antimicrobial Resistance in Livestock and Livestock Products in ASEAN Countries », Thai J Vet Med 44, n 1 (2014): 5.

131 Aude Teillant, "How much would it cost to ban antibiotic growth promoters in the livestock sector? »(3 mars 2015) <http://www.cddep.org/blog/posts/how_much_would_it_cost_ban_ antibiotic_growth_promoters_livestock_sector\#sthash.qAP2VAXn.dpbs>.

Ibid. 
davantage les choses, car les antimicrobiens «facteurs de croissance " peuvent atteindre leur objectif en assurant que les animaux ne tombent pas malades, ce qui engendrerait des problèmes de croissance. En effet, certaines juridictions font référence aux facteurs de croissance comme produits qui améliorent l'efficacité de l'alimentation des animaux.

Il devient alors difficile de tracer la ligne entre l'usage d'antimicrobiens pour stimuler la croissance et pour prévenir les maladies. Parmi les juridictions qui limitent ou interdisent les facteurs de croissance, très peu ont pris position sur l'usage préventif, qui est normalement considéré thérapeutique et bénéfique. Cependant, certains pays, comme le Danemark, ont remarqué le lien et la possibilité d'abus.

Le 17 juin 2016, l'Union européenne a adopté des conclusions par rapport aux prochaines étapes de l'approche «One Health » dans la lutte contre la résistance antimicrobienne ${ }^{133}$. Celles-ci portaient entre autres sur des cibles de réduction des infections et de la résistance antimicrobiennes chez les animaux, des actions qui auraient pour effet d'éviter le recours préventif et régulier aux antibiotiques, ainsi que sur la création de mesures pour assurer le respect des lois sur la vente et l'usage d'antimicrobiens en médecine vétérinaire. Les États membres sont également encouragés à adopter des mesures législatives plus « ambitieuses » pour combattre la résistance aux antimicrobiens, par exemple, en ce qui concerne les produits pharmaceutiques vétérinaires et la nourriture animale médicamentée. ${ }^{134}$

\subsubsection{Normes internationales}

Il existe des normes internationales qui visent à encourager l'usage judicieux des antimicrobiens, comme, par exemple, les limites maximales de résidus (LMR) fixées par le Codex Alimentarius (Codex) ${ }^{135}$. Le Codex, en tant qu'organisation internationale qui établit des normes alimentaires, des lignes directrices et des codes d'usages ${ }^{136}$, occupe une position unique pour apporter une réponse générale et multidisciplinaire aux questions soulevées par la résistance antimicrobienne chez les animaux d'élevage ${ }^{137}$. En 2005, le Codex a émis un Code d'usages visant à réduire au minimum et à maîtriser la résistance aux antimicrobiens, pour ainsi fournir des indications sur l'usage responsable et prudent des antimicrobiens pour les animaux élevés pour l'alimentation humaine. Ces indications s'ajoutent à celles d'un autre document du Codex, soit le Code d'usages international recommandé pour le contrôle de l'utilisation des médicaments vétérinaires.

Ces normes internationales peuvent guider les choix politiques des gouvernements nationaux et contribuer à l'harmonisation internationale sur certains points. Plusieurs pays en voie de développement les considèrent particulièrement utiles

133 Council of the European Union, «Council conclusions on the next steps under a One Health approach to combat antimicrobial resistance » (17 juin 2016) <http://www.consilium.europa.eu/ en/press/press-releases/2016/06/17-epsco-conclusions-antimicrobial-resistance/>. Ibid.

135 Codex Alimentarius Commission, « Maximum Residue Limits for Veterinary Drugs in Foods » (juillet 2012) <ftp://ftp.fao.org/codex/weblinks/MRL2_e_2012.pdf>.

136 Â propos du Codex Alimentarius. <http://www.fao.org/fao-who-codexalimentarius/aboutcodex/fr/ $>$.

137 Résistance aux antimicrobiens. <http://www.fao.org/fao-who-codexalimentarius/themes/ antimicrobial-resistance/fr/>. 
pour harmoniser leurs normes, obtenir de l'aide technique et atteindre les exigences de leurs partenaires commerciaux ${ }^{138}$. Il est toutefois à noter que les normes établies par le Codex ne sont pas obligatoires. Cependant, elles servent de point de référence pour l'Organisation mondiale du commerce (OMC) lors de disputes ${ }^{139}$. L'OMC, en revanche, peut imposer des sanctions par le biais de ses pays membres ${ }^{140}$.

\subsubsection{Manque soutenu de surveillance et de données}

Présentement, il y a très peu de surveillance et de données sur la quantité et l'utilisation des antimicrobiens chez les animaux d'élevage. Pourtant, la surveillance est essentielle $^{141}$ pour orienter les politiques et pour identifier où ces interventions devraient avoir lieu ${ }^{142}$. Idéalement, les pays devraient mettre en place des programmes de surveillance nationaux ${ }^{143}$. Certains pays recueillent déjà quelques données, sans pour autant recueillir suffisamment d'informations pour qu'elles puissent aider à l'élaboration de politiques. Les États-Unis et l'Australie, par exemple, recueillent seulement des informations sur le volume des ventes de médicaments antimicrobiens; des informations clés, comme qui administre les médicaments et à quels animaux, sont absentes ${ }^{144}$.

Ainsi, des politiques sur la surveillance et la collecte de données dans le milieu de la production pourraient permettre aux décideurs d'avoir une meilleure idée des problématiques propres à leur pays, pour ensuite cibler les activités et acteurs clés.

\subsection{Commerce de produits agricoles}

\subsubsection{Systèmes à double vitesse}

Les législateurs subissent l'influence d'une multitude de facteurs tels que la méthode de production, le niveau d'industrialisation, la taille des industries et leurs rapports avec le gouvernement, la capacité des autorités d'assurer la règlementation, les considérations plus larges de politique internationale, ainsi que la culture (ex. les parties de l'animal consommées). La définition de ce qui constitue l'usage judicieux d'agents antimicrobiens soulève bon nombre de points contentieux entre les juridictions.

Cependant, l'influence des grands marchés n'est pas en soi déterminante. Les interdictions dans les grands marchés d'importation n’encouragent pas nécessairement les pays exportateurs à adopter des pratiques similaires partout sur leur territoire. Plutôt, des marchés à « double vitesse » apparaissent où les règlements qui encadrent les produits destinés à la consommation domestique sont plus permissibles que ceux qui encadrent les produits destinés à d’autres marchés.

138 World Trade Organization, « Members greet food safety body's half century with plea for sciencebased trade measures " (28 juin 2013) <https://www.wto.org/english/news_e/news13_e/ sps_28jun13_e.htm>. Codex Alimentarius Commission. <https://www.food.gov.uk/about-us/codex-alimentariuscommission>.

140 Whose WTO is it anyway? <https://www.wto.org/english/thewto_e/whatis_e/tif_e/org1_e.htm>

141 Sharon Levy, "Reduced Antibiotic Use in Livestock: How Denmark Tackled Resistance ", Environmental Health Perspectives 122, nº 6 (2014): A160 <http://ehp.niehs.nih.gov/122-a160/>.

142 «Australian One Health », p. 11

143 Ibid.

144 Levy; " Antimicrobial resistance: A Report ». 


\subsubsection{Interdictions totales ou au cas par cas}

Bien que certains gouvernements interdisent des types d'usages dans leur totalité ou presque, la plupart des pays créent plutôt des interdictions au cas par cas. Il y a plus de consensus en ce qui a trait à certaines substances spécifiques. Par exemple, malgré les tensions entre les États-Unis et l'Europe à ce sujet, la grande majorité des juridictions interdisent les produits provenant de l'élevage d'animaux qui ont reçu de la ractopamine. Il est dit que 160 pays, y compris la Chine et la Russie, dont les systèmes de salubrité des aliments sont parfois critiqués, interdisent la ractopamine en raison d'inquiétudes au niveau de la santé humaine. Malgré tout, certains producteurs et exportateurs très importants sur la scène internationale, tels les États-Unis, utilisent encore cette substance ${ }^{145}$.

\subsubsection{Normes internationales}

Les normes internationales discutées dans la section précédente peuvent également agir au niveau du commerce international. La légalité de l'interdiction européenne de l'usage de la ractopamine en l'absence de preuve scientifique « concluante " peut soulever quelques questions quant à la légitimité des interdictions imposées par certains pays qui limitent l'accès à des marchés et, conséquemment, le commerce. En 2012, suite à un vote très controversé, une limite maximale de résidus (LMR) pour la ractopamine a été ajoutée au Codex ${ }^{146}$.

Effectivement, bien qu'elles n'aient pas force exécutoire, ces normes internationales peuvent limiter les recours des gouvernements individuels en vertu d'obligations internationales. Le Codex Alimentarius et l'Organisme mondial du commerce (OMC) entretiennent un lien collaboratif institutionnel. C'est-à-dire que l'OMC dépend de l'expertise du Codex ${ }^{147}$. Mais la preuve scientifique qui appuie les décisions de la Commission du Codex Alimentarius peut être grandement politisée ${ }^{148}$. En 2012, la Commission du Codex Alimentarius a adopté une LMR pour la ractopamine $^{149}$, ce qui va à l'encontre de plusieurs pays avec une politique de «tolérance zéro » quant à l'utilisation de cette substance ${ }^{150}$.

Ces normes internationales peuvent être attrayantes pour les pays qui veulent adopter un modèle préétabli et qui se fient à l'expertise technique du $\operatorname{Codex}^{151}$. Mais la légalité de ces interdictions a depuis longtemps été questionnée auprès de l'OMC. Depuis le cas sur la désignation commerciale des sardines ${ }^{152}$, soumis à

145 Wayne Pacelle, « Banned in 160 Nations, Why is Ractopamine in U.S. Pork? (Op-Ed) » (26 juillet 2014) <http://www.livescience.com/47032-time-for-us-to-ban-ractopamine.html>.

146 Shefali Sharma, « 10 reasons TTIP is bad for good food and farming » (2014) <http://www.iatp. org/files/2014_05_16_10ReasonsTTIP_SS.pdf]>.

147 Alberto Alemanno et Giuseppe Capodieci, « Testing the Limits of Global Food Governance: The Case of Ractopamine » (2013), p. $11<\mathrm{http} / /$ www.hec.fr/var/corporate/storage/original/application/ 5dae2fce5d311b02f58675c03c946aed.pdf $>$.

148 Alemanno et Capodieci.

149 Codex Alimentarius Commission.

150 Alemanno et Capodieci, p. 2.

151 Ibid., p. 9.

152 Communautés européennes - Désignation commerciale des sardines, Règlement des différends: affaire DS231, Organisation Mondiale du Commerce <https://www.wto.org/french/tratop_f/ dispu_f/cases_f/ds231_f.htm>. 
l'organe de règlement des différends de l'OMC, il est établi que les pays membres de l'OMC peuvent être liés par des normes internationales, même si celles-ci sont volontaires et que le pays en question a voté contre cette norme.

Ces considérations indiquent qu'il existe un degré de fragmentation au sein de la gouvernance mondiale des aliments ${ }^{153}$. Celle-ci crée de l'incertitude sur le plan juridique, d'où les disputes entre les grands marchés et les retards quant à l'adoption de nouveaux accords commerciaux.

\section{Passer à l'action}

La lutte contre la résistance antimicrobienne requiert des efforts concertés entre plusieurs acteurs. ${ }^{154}$ Tel que suggéré par l'OMS, les pays doivent assurer la coordination et la collaboration de tous les secteurs et acteurs concernés, tant au plan horizontal, entre les gouvernements et les autres organismes, qu'au plan vertical, entre les différents niveaux de gouvernement et les diverses strates parmi les secteurs ${ }^{155}$. Bien qu'il soit impératif que les gouvernements passent à l'action, ils ne peuvent résoudre ce problème à eux seuls : ils devront s'assurer la participation de tous les acteurs concernés, dont les groupes professionnels, le secteur privé et les sociétés civiles, afin d'atteindre leurs objectifs ${ }^{156}$.

À la lumière des conclusions de cette étude, des incitations, ainsi que des obstacles qui peuvent jouer un rôle déterminant en matière de politiques ont été identifiés.

\subsection{Incitations}

\subsubsection{Incitation $n^{o} 1$ : la santé publique}

Bien que différents acteurs ou juridictions décident d'agir pour différentes raisons, les impératifs de santé humaine entrent toujours dans l'équation. Dans certains pays, ceci est le jalon utilisé pour marquer la distinction entre les utilisations permises et interdites d'antimicrobiens.

Des listes d'antimicrobiens importants en santé humaine sont souvent utilisées à cette fin. Certaines juridictions qui tardent à agir ou qui n'ont adopté que des mesures volontaires, comme par exemple les États-Unis, ${ }^{157}$ les utilisent. Dans d'autres pays, comme le Japon ${ }^{158}$, ces listes sont utilisées dans le cadre du processus de gestion du risque lors de l'approbation de nouveaux médicaments vétérinaires.

La disponibilité de données et d'études sur l'utilisation et les effets des antimicrobiens est aussi d'une très grande importance. Dans bien des cas, il peut être

\footnotetext{
153 Alemanno et Capodieci, p. 13.

154 Tackling antimicrobial resistance (AMR) together. Working paper 1.0: Multisectoral coordination. Geneva: World Health Organization; 2018 (WHO/HWSI/AMR/2018.2). <http://www.who. int/antimicrobial-resistance/publications/Tackling-AMR-multisectoral-coordination-june2018. pdf>

155 Ibid., p. 1

156 Ibid.

157 Animal Health Institute, «Animal Antibiotics: Keeping Animals Healthy and Our Food Safe » $<$ http://www.ahi.org/wp-content/uploads/2011/04/Animal-Antibiotics-Keeping-AnimalsHealthy.pdf $>$.

158 National Veterinary Assay Laboratory, p. 19.
} 
nécessaire de démontrer un lien entre l'utilisation d'un agent antimicrobien et des conséquences néfastes pour convaincre les législateurs d’agir.

Au niveau pratique, des différences culturelles jouent également à cet égard. Dans certains marchés, différentes parties du corps animal sont consommées. Puisque l'effet des médicaments sera différent, par exemple, sur le foie et sur les muscles, des marchés comme la Chine ne permettent pas l'usage de certains antibiotiques chez certains animaux (par exemple, le sulfathiazole chez le porc) et établissent des limites plus basses pour les résidus d'autres médicaments (par exemple, la tétracycline et l'oxytétracycline) ${ }^{159}$.

\subsubsection{Incitation $n^{\circ} 2$ : la compétitivité commerciale}

Les marchés qui prennent du retard en matière de réglementation auront de plus en plus de difficulté à conserver leur rôle sur le marché mondial, surtout lorsque la demande de produits de plus haute qualité ou dont la qualité peut être garantie devient une exigence ${ }^{160}$. Les interdictions portant sur les facteurs de croissance sont de plus en plus répandues. Compte tenu du fait que ces changements réglementaires doivent être introduits progressivement, les retards pourraient vite s'accumuler dans les marchés qui nont pas encore une stratégie pour passer à l'action. La réglementation des antimicrobiens peut donc être considérée comme une occasion de préserver ou d’accroître la compétitivité des marchés.

\subsubsection{Incitation $n^{o} 3$ : les conséquences de l'inaction}

Somme toute, sans règles supplémentaires pour encadrer l'utilisation des antimicrobiens dans le domaine de l'agriculture, la consommation mondiale d'antimicrobiens chez les animaux délevage pourrait augmenter de $67 \%$ ou plus d'ici $2030^{161}$. La Chine et l'Inde, deux pays qui n'ont que très peu ou aucun règlement sur les antimicrobiens, contribueront grandement à cette augmentation ${ }^{162}$. Ces chiffres indiquent qu'avec l'inaction réglementaire, les risques de dérapage ne sont pas à ignorer.

\subsubsection{Incitation $n^{\circ} 4$ : les changements dans l'industrie domestique}

Les États-Unis sont parmi les pays les plus industrialisés qui nont toujours pas mis en place des règles ambitieuses pour réduire de façon importante la consommation d'antimicrobiens à des fins autres que thérapeutiques. Certaines mesures sont promises, mais, concrètement, peu de progrès ont été effectués sur le plan règlementaire. Bien que le congrès américain tarde à agir, déjà, de grandes chaînes de restauration ont choisi de modifier leurs pratiques et d'offrir à leurs clients de la viande produite sans facteurs de croissance ${ }^{163}$. Des grands producteurs de viande

159 USDA Food Safety and Inspection Service, « Export Requirements for The People’s Republic of China » (25 avril 2016) <http://www.fsis.usda.gov/wps/portal/fsis/topics/international-affairs/ exporting-products/export-library-requirements-by-country/Peoples-Republic-of-China $>$.

161 Van Boeckel.

162 Ibid.

163 P. Collignon, « Antibiotic resistance: are we all doomed? », Internal Medicine Journal 45, $\mathrm{n}^{\circ} 11$ (2015): $1109<$ http://onlinelibrary.wiley.com/enhanced/doi/10.1111/imj.12902/>. 
ont aussi cessé d'utiliser des antibiotiques d'importance en santé humaine comme facteurs de croissance et à des fins préventives. Au Danemark, l'industrie avait également choisi de changer ses pratiques avant même que le législateur ne crée des interdictions.

L'industrie peut ainsi simplifier la tâche du législateur, puisque des leçons importantes ressortiront certainement de la mise en œuvre de ces nouvelles pratiques. De plus, des producteurs pourraient être davantage incités à agir si des acheteurs importants exigeaient des produits qui respectent certaines normes.

\subsection{Obstacles}

\subsubsection{Obstacle $n^{0} 1$ : le manque de mesures scientifiques précises}

Le manque de mesures précises et universellement acceptées dans les études scientifiques portant sur les antimicrobiens est un obstacle à la réglementation. Bien que l'utilisation d'antibiotiques chez les animaux d'élevage comporte des risques pour la santé humaine, une incertitude perdure quant aux conséquences spécifiques ${ }^{164}$. Des acteurs dont les intérêts ne sont pas alignés avec les impératifs de santé publique peuvent utiliser l'incertitude pour convaincre les décideurs de retarder l'adoption de mesures réglementaires fondées sur des données scientifiques valables. Cette dynamique pourrait encourager une approche qui interdise des antimicrobiens spécifiques plutôt que la réglementation d'une catégorie ou d'un type d'utilisation dans son entièreté, puisqu'il sera plus facile d'apporter une preuve scientifique concluante pour un produit en particulier plutôt qu'une catégorie de produits ou un type d'utilisation.

\subsubsection{Obstacle $n^{0} 2$ : les pressions politiques et commerciales}

Des groupes d'intérêts puissants dans un pays peuvent retarder l'adoption de mesures qui font pourtant l'objet d'un consensus presque unanime. Par exemple, au Canada, la faille réglementaire qui permet l'importation de produits qui sont autrement interdits s'ils sont destinés à l'utilisation personnelle est bien connue. Des associations professionnelles, tel l'Institut canadien de la santé animale ${ }^{165}$, ainsi que des membres de la profession vétérinaire ${ }^{166}$, aimeraient que le législateur agisse. Cependant, d'autres organisations, telle la Canadian Cattlemen's Association, veulent des preuves scientifiques supplémentaires ${ }^{167}$.

Pareillement, les partenaires commerciaux et la division des marchés peuvent influencer les décideurs. En mars 2017, la Chine, qui refusait toujours de lever son embargo sur les produits français à la suite de la crise de la vache folle en 2001,

164 Timothy F. Landers et al., "A Review of Antibiotic Use in Food Animals: Perspective, Policy, and Potential » (2012) 127 Public Health Reports 4 <http://www.ncbi.nlm.nih.gov/pmc/articles/ PMC3234384/pdf/phr12700004.pdf>.

165 Susan Mann, «Health Canada proposes tighter controls over veterinary drug imports » (22 avril 2015) <http://www.betterfarming.com/online-news/health-canada-proposes-tighter-controls-overveterinary-drug-imports-60800>.

166 College of Veterinarians of Ontario.

167 Aruna Handa et Paul Webster, «Industry-led committee urges delay in closing loophole allowing import of unapproved antibiotics for animals », Canadian Medical Association Journal 180, $\mathrm{n}^{\circ} 9$ (2009): 914-916 <http://www.cmaj.ca/content/180/9/914.full>. 
a accepté d’assouplir les mesures restrictives imposées à la viande de bœuf française, sous certaines conditions dont un audit du système sanitaire français ${ }^{168}$. Il a été suggéré que ce changement s'effectuait en réaction à des réalités politiques, la Chine étant soucieuse de renforcer ses liens avec l'Union européenne pour contrer les effets du protectionnisme américain sous l'administration Trump ${ }^{169}$.

\subsubsection{Obstacle $n^{\circ} 3$ : l'insuffisance des infrastructures}

Le plus grand obstacle auquel se confronte la réglementation peut bien être la mise en œuvre. Par exemple, le gouvernement chinois a adopté quelques règles sur l'usage des antimicrobiens, mais, en raison de lacunes au niveau de l'infrastructure, plusieurs manquements sont rapportés ${ }^{170}$. Les modes de production et l'organisation de la production peuvent également créer des problèmes. En aquaculture, par exemple, certains producteurs ne sont pas enregistrés et échapperaient aux mesures de contrôle ${ }^{171}$.

Au Canada, malgré une infrastructure assez développée, plusieurs antimicrobiens échappent au contrôle réglementaire. Le système prévoit des exceptions réglementaires pour l'utilisation personnelle, ce qui crée une faille au niveau de la surveillance. Une surveillance plus accrue permettrait au législateur canadien d'imposer les médicaments vétérinaires pour réduire la consommation de produits antimicrobiens. Des études ont démontré qu'en médecine humaine, il existe un lien entre le coût d'un médicament et la fréquence à laquelle il est prescrit. Le même principe pourrait agir en médecine vétérinaire ${ }^{172}$. Cette approche encouragerait également les fermiers à tenir dûment compte des traitements autres que les antimicrobiens ${ }^{173}$. Mais, pour que cette mesure réglementaire soit réellement efficace, des données additionnelles sont nécessaires pour comprendre ce qui motive les choix des vétérinaires et leur usage des antimicrobiens ${ }^{174}$.

De plus, il se peut qu'il existe une corrélation plus forte entre la corruption et la résistance antimicrobienne qu'entre l'utilisation d'antimicrobiens et la résistance qui en découle ${ }^{175}$. Ces résultats illustrent bien la nécessité de prévoir un système réglementaire étanche avec des mécanismes de contrôle et de surveillance pour assurer que les exigences réglementaires soient respectées.

\section{Conclusion}

Une tendance principale se dégage de cette étude : les pays industrialisés dont les marchés sont bien établis tendent à adopter des règlements qui agissent plus tôt

\footnotetext{
168 Antoine d'Abbundo, « Pékin assouplit l'embargo sur le bœuf français », LaCroix (3 mars 2017) <http://www.la-croix.com/Economie/France/Pekin-assouplit-lembargo-boeuf-francais-201703-05-1200829433>.

169 Ibid.

170 Broughton et Walker.

171 Ibid.

172 «Australian One Health », p. 13.

173 Jim O’Neill, " Antimicrobial in Agriculture and the Environment: Reducing Unnecessary Use and Waste »(2015), p. 29 <http://amr-review.org/sites/default/files/Antimicrobials\%20in\%20 agriculture $\% 20$ and $\% 20$ the $\% 20$ environment $\% 20$-\%20Reducing $\% 20$ unnecessary $\% 20$ use $\% 20$ and $\% 20$ waste.pdf $>$.

175 Collignon.
} 
dans le processus agricole, une approche qui agit à la source, alors que les pays en voie de développement, dont les marchés sont en pleine évolution, tendent plutôt à opter pour des mécanismes réglementaires régissant le commerce des produits agricoles, une approche axée sur les produits finis. Bien qu'un système réglementaire qui agit à la source puisse être très efficace, il est bien probable qu'il serait confronté à des obstacles de taille lors de la mise en ouvre dans certains contextes. Par exemple, des mesures qui encadrent étroitement les médicaments vétérinaires en exigeant une ordonnance au point de vente seront peu utiles dans un pays où il existe une pénurie de vétérinaires. De plus, si, dans ce même pays, les antimicrobiens sont utilisés pour pallier des lacunes au niveau de la salubrité des aliments, cette nouvelle exigence risquerait alors d’avoir des impacts négatifs sur la santé publique.

Ceci dit, linaction face à l'augmentation de la résistance antimicrobienne aurait aussi de graves conséquences. Les décideurs sont donc confrontés à des défis de taille en matière de réglementation des antimicrobiens. Ils doivent pouvoir identifier les mesures appropriées à leur contexte, compte tenu des ressources disponibles, de l'infrastructure existante et des intérêts qui gouvernent les industries concernées, afin d’assurer leur participation. De plus, le commerce international peut rapidement prendre un ton politique qui risque déclipser d’autres considérations (par exemple, en Russie).

Dans tous les cas, peu importe l'approche retenue, les antimicrobiens encadrent trois points d'entrée critiques : la médecine vétérinaire, la production agricole et le commerce. Il s'agirait alors d'une approche multidisciplinaire qui dépendra grandement de la pleine participation de plusieurs acteurs.

Une étude comparative et systématique détaillée des exigences techniques établies par les différents systèmes réglementaires discutés permettrait d’approfondir cette discussion. De plus, au fur et à mesure que ces données deviendront disponibles, il serait important de mesurer le taux de succès des approches discutées pour tirer davantage de conclusions qui vont au-delà de la portée de cette étude.

Prativa Baral

Global Strategy Lab, Dahdaleh Institute for Global Health Research

Faculty of Health and Osgoode Hall Law School

York University, Toronto

prativa.baral@globalstrategylab.org

Marie E. Danik

Association des juristes d'expression

française de l'Ontario, Ottawa

mdanik@ajefo.ca

Steven J. Hoffman

Global Strategy Lab, Dahdaleh Institute for Global Health Research

Faculty of Health and Osgoode Hall Law School

York University, Toronto

steven.hoffman@globalstrategylab.org 